J. Noncommut. Geom. 7 (2013), 709-735

DOI $10.4171 / \mathrm{JNCG} / 132$
Journal of Noncommutative Geometry

(C) European Mathematical Society

\title{
An equivariant noncommutative residue
}

\author{
Shantanu Dave*
}

\begin{abstract}
Let $\Gamma$ be a finite group acting on a compact manifold $M$ and let $\mathcal{A}(M)$ denote the algebra of classical complete symbols on $M$. We determine all traces on the cross-product algebra $\mathcal{A}(M) \rtimes \Gamma$ as residues of certain meromorphic zeta functions. Further we compute the cyclic homology for $\mathcal{A}(M) \rtimes \Gamma$ in terms of the de Rham cohomology of the fixed point manifolds $S^{*} M^{g}$. In the process certain new results on the homologies of general crossproduct algebras are obtained.
\end{abstract}

Mathematics Subject Classification (2010). 58J42; 19D55,58J40.

Keywords. Noncommutative residue, cyclic homology, cross-product algebra.

\section{Introduction}

On a closed manifold $M$ the (classical) pseudo-differential operators form an algebra $\Psi^{\infty}(M)$. The space of smoothing operators $\Psi^{\infty}(M)$ is then an ideal and the quotient $\mathcal{A}(M):=\Psi^{\infty}(M) / \Psi^{-\infty}(M)$ is called the algebra of complete symbols. Let $\Gamma$ be a finite group acting on $M$ by diffeomorphisms. The group $\Gamma$ then acts on $\Psi^{\infty}(M)$ and on $\mathcal{A}(M)$ by push-forward of operators, namely if $\mathscr{D}$ is a pseudo-differential operator and $g \in \Gamma$, then

$$
g \cdot \mathcal{D}(f):=g \mathscr{D}\left(g^{-1} f\right) \text { for all } f \in \mathcal{C}^{\infty}(M) .
$$

In this paper we consider traces on the cross-product algebra $\mathcal{A}(M) \rtimes \Gamma$ and compute its Hochschild and cyclic homology groups. We consider some applications to asymptotics of representations.

The traces on $\mathcal{A}(M) \rtimes \Gamma$ are considered as equivariant generalization of the noncommutative residue of Wodzicki [27] and Guillemin [12]. To a generator $A g$ in $\mathcal{A}(M) \rtimes \Gamma$ and an invariant order 1 positive elliptic operator $D$ one associates the zeta-function,

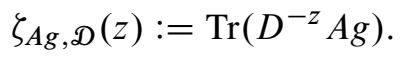

By means of stationary phase analysis near the fixed points of the diffeomorphism $g$, a meromorphic extension of these zeta functions to the whole of $\mathbb{C}$ with some

\footnotetext{
* Supported by FWF grant Y237-N13 of the Austrian Science Fund.
} 
simple poles is provided. Then for a fixed conjugacy class $\langle\gamma\rangle$ a trace on $\mathcal{A}(M) \rtimes \Gamma$ is obtained by

$$
\operatorname{Tr}_{\langle\gamma\rangle}\left(\sum_{g \in \Gamma} A_{g} g\right):=\operatorname{res}_{z=0}\left(\sum_{g \in\langle\gamma\rangle} \zeta_{A g, D}(z)\right),
$$

which we shall refer to as equivariant noncommutative residue associated to the conjugacy class $\langle\gamma\rangle$.

The above mentioned trace has applications analogous to the noncommutative residue. For instance we obtain a very special case of an equivariant Weyl's formula of [5], namely if $\Gamma$ acts faithfully on $M$ and $\pi$ is an irreducible representation of $\Gamma$, then, for an invariant operator $\mathscr{D}$, with eigenvalues $\lambda_{i}$ and corresponding eigenspaces $V_{i}$,

$$
N_{\pi, D}(\lambda):=\sum_{\lambda_{i}<\lambda} \text { "multiplicity of } \pi \text { in } V_{i} " \simeq \frac{C}{\operatorname{dim} \pi} \lambda^{\frac{\operatorname{dim} M}{\operatorname{order}(D)}} .
$$

This technique can be applied to the difference of certain representations $\pi_{1}-\pi_{2}$ enabling a study of comparative asymptotics of representations. Further results include an equivariant Connes trace formula as well as extensions of the logarithmic symbols based on a 2 cocycle in $H^{2}\left(S_{\log }(M) \rtimes \Gamma\right)$ as in [13].

Hence the question arises whether the traces defined in (1) are all the traces that can arise on $\mathcal{A}(M) \rtimes \Gamma$. By computing the homologies of $\mathcal{A}(M) \rtimes \Gamma$ we shall find an answer to this question as well as understand the higher analogues of these equivariant noncommutative residues.

Our result is as follows. Let $\Gamma_{\gamma}:=\{g \in \Gamma \mid g \gamma=\gamma g\}$ be the centralizer of $g$ in $\Gamma$. Let $k_{\gamma}=\operatorname{dim}\left(T^{*} M^{\gamma}\right)$. Then

$$
\mathrm{HH}_{k}(\mathcal{A}(M) \rtimes \Gamma)=\sum_{\langle\gamma\rangle} H^{k_{\gamma}-k}\left(S^{*} M^{\gamma} \times S^{1}\right)^{\Gamma_{\gamma}} .
$$

Here the sum is taken over a set of representatives of the conjugacy classes. Also

$$
\mathrm{HC}_{k}(\mathcal{A}(M) \rtimes \Gamma)=\sum_{j \geq 0} \mathrm{HH}_{k-2 j}(\mathcal{A}(M) \rtimes \Gamma) .
$$

Our determination of these homology groups extends the results of [7], using also techniques from [8], [9]. Interestingly, there are some qualitatively new phenomena arising at the nontrivial conjugacy classes that are not expected from the non-equivariant case.

The cross-product algebra $\mathcal{A}(M) \rtimes \Gamma$ has a natural filtration that comes from the order of the operators on $\mathcal{A}(M)$. We use the spectral sequence associated to this filtration in our homological computation. The first hurdle here is that the associated graded algebra $\operatorname{Gr}(\mathcal{A}(M)) \rtimes \Gamma$ is noncommutative. Nevertheless, this algebra is the cross-product of a commutative algebra by a finite group, and as such it preserves many features of commutativity. In particular, its Hochschild homology has a description using differential forms on the fixed point sets of the elements of the group [3]. The 
differentials in the spectral sequence turn out to preserve this structure. The action of the first relevant differential, $d_{2}$, is similar to the one in the case without group action as explained in [7], albeit technically different. Here one can exploit the structure of certain symplectic submanifolds of the cotangent bundle. This explains the fact that the residue trace associated to each conjugacy class of $\Gamma$ (provided that that conjugacy class has a nonempty, connected fixed point set) will no longer have the property of being localized to a singly homogeneous component of the symbol in any coordinate neighborhood. This is in contrast to the usual case when there is no group action, where the residue trace is localized on the component of homogeneity $-n$ of the complete symbol.

As mentioned above, we need to know as explicitly as possible the Hochschild homology groups of $\complement^{\infty}\left(S^{*} M\right) \rtimes \Gamma$. These homology groups are already well known from the results of Baum and Connes [3] on the homology of cross-products by proper actions or from [8]. However in Sections 4 and 5 of this paper we provide a more mundane and explicit calculation which we shall need in order to identify the terms in the above mentioned spectral sequence. Sections 6 and 7 give the necessary technical background in topologically filtered algebra and symplectic geometry. The main homology result is computed in Section 8. Further the paper is organized as follows. We begin in Section 2 with the analysis of the zeta functions $z \mapsto \operatorname{Tr}\left(D^{-z} A g\right)$ by the use of the stationary phase principle. In Section 3, we use the Tauberian theorem to obtain some results on asymptotics of representations in eigenspaces.

In [10] an abstract algebra of pseudo-differential operators is associated to a spectral triple. The cross-product $\mathcal{A}(M) \rtimes \Gamma$ is the algebra of abstract pseudo-differential operators associated to the spectral triple of the crossed-product algebra $\bigodot^{\infty}(M) \rtimes \Gamma$ in case of an equivariant Dirac operator on a spin manifold. ${ }^{1}$ The current work is partly motivated by the desire to understand abstract pseudo-differential operator algebras. Furthermore we also expect to use the cyclic homology computations in this paper to obtain another perspective on the Lefschetz fixed point formula in [2] using an excision argument in cyclic homology [28].

Acknowledgment. I would like to thank Victor Nistor for all his suggestions and comments. I would like to also thank Matthias Lesch for pointing me to the work of Brüning [5] on computation of asymptotics of representations of eigenspaces on invariant operators.

\section{Explicit description of traces}

Here we shall construct a nontrivial trace on the cross-product algebra $\mathcal{A}(M) \rtimes \Gamma$, corresponding to those conjugacy classes $\langle\gamma\rangle$ of the group $\Gamma$, so that the elements $\gamma \in\langle\gamma\rangle$ have a nonempty fixed point set on the cosphere bundle, that is, $S^{*} M^{\gamma} \neq \emptyset$.

\footnotetext{
${ }^{1}$ The computations in this paper extend to the case of group actions on vector-bundles and the corresponding cross-product of pseudo-differential operators.
} 
The homology computations of latter sections show that under suitable conditions these are all the traces on $\mathcal{A}(M) \rtimes \Gamma$.

Let us fix our terminology. In the sequel a constant order holomorphic family $A(z) \in \Psi^{m}(M)$ of pseudo-differential operators on a closed manifold $M$ is one that can be obtained from a holomorphic family of complete symbols of fixed order and a holomorphic family of regularizing operators. (The regularizing operators are given the Fréchet topology of $\mathcal{C}^{\infty}(M \times M)$.) Furthermore, by a holomorphic family $A(z) \in \Psi^{z}(M)$ we mean that $A(z)=B(z) D^{z}+C(z)$, where $B$ is a holomorphic family of order-zero operators, $D$ is a positive first order pseudo-differential operator, and $C(z)$ is a holomorphic family of regularizing operators. See [1], [15], [16], [21] and the references therein for more details.

Let us fix a positive, elliptic, order one operator $\mathscr{D} \in \Psi^{1}(M)$ that is invariant under the $\Gamma$ action. This is to say $\mathscr{D} g=g \mathscr{D}$ as operators on $\mathcal{C}^{\infty}(M)$ for all $g \in \Gamma$. The complex powers $\mathscr{D}^{z}$ can be defined easily by the spectral theorem, but it is a more subtle fact that each $\mathscr{D}^{z}$ is a pseudo-differential operator of order $z$. This was proved by Seeley [26]. Another proof was given by Guillemin in [12].

In particular, Seeley's result implies that for $\operatorname{Re}(z)<-n$, the operator $\mathfrak{D}^{z}$ is of trace-class, and hence the map

$$
z \rightarrow \operatorname{tr}\left(\mathscr{D}^{z}\right)
$$

is a holomorphic function on the half plane $\operatorname{Re}(z)<-n$ of complex numbers.

For $A$ in $\Psi^{\infty}(M)$ and any group element $g$, we define

$$
\zeta_{g, A}(z):=\operatorname{Tr}\left(\mathscr{D}^{-z} A g\right) .
$$

This function is a priori defined only when $D^{-z} A$ is a trace-class operator, which is the case when $\operatorname{Re}(z)>n+\operatorname{order}(A)$. A standard stationary phase argument applied near the fixed point submanifold $M^{g}$ provides the following result (see [11]).

Proposition 2.1. Let $k_{g}=\operatorname{dim} M^{g}, \mathscr{D}$ a positive order one elliptic operator invariant under $\Gamma$ and $A$ any classical pseudo-differential operator. Then the function $z \mapsto \operatorname{Tr}\left(D^{-z} A g\right)$ is holomorphic on the half plane $\operatorname{Re}(z)>d, d=k_{g}+\operatorname{order}(A)$, and has a meromorphic extension with possible simple poles at $z=d, d-1$, $d-2, \ldots$.

In fact in Proposition 2.1, the operators $\mathscr{D}^{-z} A$ could be replaced by any holomorphic family $R(z)$ with order $R(z)=-z$.

Remark 2.2. For any order $m$ positive operator $D$, the operator $\mathscr{D}=D^{\frac{1}{m}}$ is of order one and hence for the operator $D$ its zeta function $\zeta_{g, A}(z)=\operatorname{Tr}\left(D^{-z} A g\right)$ has a meromorphic extension to the complex numbers with possible poles at $\frac{d}{m}, \frac{d-1}{m}, \ldots$.

It is for example known that nonsimple poles can occur in the zeta function of operators on spaces with singularities [24], [17], [21]. 
Although the function $\zeta_{g, A}$ does depend on the choice of an invariant operator $\mathscr{D}$, the residue of $\zeta_{A, g}$ at $z=0$ does not. Indeed if $\mathscr{D}_{1}$ is another such operator invariant under $\Gamma$, then let $R(z)=\mathscr{D}_{1}^{-z}-\mathscr{D}^{z}$. Since $R(0)=0$ there is a holomorphic family $S(z)$ such that $R(z)=z S(z)$. Thus we obtain

$$
\operatorname{res}_{z=0} \operatorname{Tr}\left(D^{-z} A g-D_{1}^{-z} A g\right)=\operatorname{res}_{z=0} \operatorname{Tr}(z S(z) A g)=0,
$$

where the last equality holds because $S(z) A g$ can have at most simple poles. Thus we can define $\tau_{g}(A):=\operatorname{res}_{z=0} \operatorname{Tr}\left(\mathscr{D}^{-z} A g\right)$ for any operator $\mathscr{D}$ with the aforementioned properties. It is immediate from the definition that $\tau_{g}$ descends to a function on $\mathcal{A}(M)$.

Let us first note that we can now indeed define traces on the cross-product of the complete symbol algebra.

Corollary 2.3. Let $\mathscr{B}(M)=\mathcal{A}(M) \rtimes \Gamma$. For any conjugacy class $\langle\gamma\rangle \in\langle\Gamma\rangle$, the map $\operatorname{Tr}_{R}^{\langle\gamma\rangle}: \mathscr{B}(M) \mapsto \mathbb{C}$ given by

$$
\operatorname{Tr}_{R}^{\langle\gamma\rangle}\left(\sum_{g \in \Gamma} A_{g} g\right):=\sum_{g \in\langle\gamma\rangle} \tau_{g}\left(A_{g}\right)
$$

is a trace on $\mathscr{B}(M)$.

Proof. By linearity it suffices to check that each $\operatorname{Tr}_{R}^{\langle\gamma\rangle}$ satisfies the trace property on the generators. If $g, h \in \Gamma$ then $g h$ and $h g$ belong to same conjugacy class and thus

$$
\tau_{\langle g h\rangle}[A g, B h]=\operatorname{res}_{z=0} \operatorname{Tr}\left(D^{-z}[A g, B h]\right) .
$$

For any operators $A, B, C$ such that $A B C, A C B$ and $B A C$ are trace class and $\operatorname{Tr}(A C B)=\operatorname{Tr}(B A C)$ the following obviously holds:

$$
\operatorname{Tr}(A[B, C])=\operatorname{Tr}([A, B] C) .
$$

Thus for $\operatorname{Re}(z)$ large enough,

$$
\operatorname{Tr}\left(\mathscr{D}^{-z}[A g, B h]\right)=\operatorname{Tr}\left(\left[\mathscr{D}^{-z}, A g\right] B h\right)=\operatorname{Tr}\left(\left[\mathscr{D}^{-z}, A\right] g B h\right) .
$$

Here the equalities hold because $\mathscr{D}$ commutes with $g$. But then $\left[\mathscr{D}^{-z}, A\right]=z R(z)$ for some holomorphic family $R(z)$. As mentioned already, $\operatorname{Tr}(R(z) g B h)$ can at most have a simple pole at $z=0$, and hence $\operatorname{res}_{z=0} z R(z) g B h=0$.

It is clear from the stationary phase analysis of Proposition 2.1 that $\operatorname{Tr}_{R}^{\langle\gamma\rangle}$ vanishes when elements of $\langle\gamma\rangle$ have no fixed points on the cosphere bundle $S^{*} M$. Let us also note when these traces are nontrivial.

Lemma 2.4. Let $m=-k_{g}$, and let $A$ be of order $m$ with principal symbol $a_{m}=$ $\sigma(A)$. Then there exists a constant $C>0$ such that $\tau_{g}(A)=C \int_{S^{*} M^{g}} \sigma(A) d \mathrm{vol}$.

In particular, if $S^{*} M^{g} \neq \emptyset$ then $\operatorname{Tr}_{R}^{\langle g\rangle}\left(D^{-k_{g}} g\right) \neq 0$ for a positive invariant order one operator $\mathfrak{D}$. 
Remark 2.5. We shall see in 8.2 that essentially all the traces on the algebra of complete symbols $\mathcal{A}(M) \times \Gamma$ are of the from $\operatorname{Tr}_{R}^{\langle\gamma\rangle}$. In general, there are some more traces on the algebra $\Psi^{\infty}(M) \times \Gamma$ which arise at isolated fixed points of elements of $\Gamma$. These traces do not descend to the complete symbol algebra as isolated fixed points do not contribute to the residues of the zeta functions. A study of traces on $\Psi^{\infty}(M) \times \Gamma$ and their relation to the Lefschetz fixed point theorem in [2] is currently in progress.

\section{Asymptotics for representations in eigenspaces}

Let $\pi \in R(\Gamma)$ be a representation of the group $\Gamma$. We can define a trace on the cross-product algebra $\mathscr{B}(M)$ corresponding to $\pi$ by

$$
\tau_{\pi}\left(\sum_{g \in \Gamma} A_{g} g\right):=\sum_{g \in \Gamma} \overline{\chi_{\pi}(g)} \tau_{g}\left(A_{g}\right) .
$$

Since $\chi_{\pi}$ is a class function, this trace is a linear combination of the traces $\operatorname{Tr}_{R}$ defined before. Let us denote by $\epsilon:=\frac{1}{|\Gamma|} \sum_{g \in \Gamma} g$ the idempotent in $\mathbb{C}[\Gamma]$. Then one can likewise obtain traces on the invariant algebra $\mathcal{A}(M)^{\Gamma}$ by $A \rightarrow \tau_{\pi}(A \epsilon)$. Such a trace is the residue of a $\zeta$ function, namely the one obtained from the meromorphic extension using Proposition 2.1 of

$$
\zeta_{\pi, A}(s):=\frac{1}{|\Gamma|} \sum_{g \in \Gamma} \overline{\chi_{\pi}(g)} \operatorname{Tr}\left(\mathscr{D}^{-s} A g\right), \quad \operatorname{Re}(s)>n .
$$

Here $\mathscr{D}$ is a $\Gamma$ invariant positive order one operator.

We say that the action of $\Gamma$ on $M$ is faithful or effective if for any $g \in \Gamma$ such that the fixed point manifold $M^{g}$ equals $M$ it follows that $g$ must be the identity element.

Lemma 3.1. Let $n=\operatorname{dim} M$. If the action of $\Gamma$ on $M$ is effective, then $\zeta_{\pi, \mathrm{Id}}$ has a pole at $s=n$.

Proof. Let $k_{g}=\operatorname{dim} M^{g}$ for any group element $g$. If the action of the group $\Gamma$ is effective then $k_{g}<n$ whenever $g$ is not the identity element $e$. Since $\zeta_{e \text {,Id }}(s)$ does have a pole at $s=n$ and all the $\zeta_{g \text {,Id }}$ do not, $\zeta_{\pi \text {,Id }}$ is holomorphic in the half plane $\operatorname{Re}(s)>n$ and must have a pole at $s=n$.

For the remainder of this section, we assume that the $\Gamma$ action is effective. Let $\left\{\lambda_{i}\right\}$ be the set of eigenvalues of $\mathscr{D}$. Then each eigenspace $V_{\lambda_{i}}=\operatorname{ker}\left(\mathscr{D}-\lambda_{i}\right)$ is invariant under $\Gamma$, and so acquires a representation $\pi V_{\lambda_{i}}$ of $\Gamma$. We count the multiplicity of the representation $\pi$ asymptotically in the eigenspaces $V_{\lambda_{i}}$. Let $\pi$ be an irreducible representation and let

$$
N_{\pi, \mathscr{D}}(\lambda):=\sum_{\lambda_{i} \leq \lambda}\left\langle V_{\lambda_{i}}, \pi\right\rangle=\sum_{\lambda_{i} \leq \lambda} \frac{1}{|\Gamma|} \sum_{g \in \Gamma} \overline{\chi_{\pi}(g)} \chi_{V_{\lambda_{i}}}(g) .
$$


The following result on the asymptotics of $N_{\pi, \mathscr{D}}(\lambda)$ is a special case of results in [5].

Proposition 3.2 (Asymptotic estimates). Let the group action of $\Gamma$ on $M$ be faithful. Then for an irreducible representation $\pi$ and $a \Gamma$ invariant operator $D$, the multiplicity counting function, $N_{\pi, \mathscr{D}}(\lambda) \sim C \operatorname{dim} \pi \lambda^{n / m}$, where $C>0$ and $m=\operatorname{ord}(\mathscr{D})$.

Proof. First we notice that for sufficiently large values of $\operatorname{Re}(s)$, the zeta functions have a nice expression of the form

$$
\zeta_{g, \mathrm{Id}}(s)=\sum_{i} \chi V_{\lambda_{i}}(g) \lambda_{i}^{-s}, \quad \operatorname{Re}(s)>k_{g} .
$$

Therefore,

$$
\zeta_{\pi, \mathrm{Id}}(s)=\int_{0}^{\infty} \lambda^{-s} d N_{\pi, \mathscr{D}}(\lambda) .
$$

By Lemma 3.1 the function $\zeta_{\pi, \text { Id }}(s)$ is holomorphic on the half plane $\operatorname{Re}(s)>n$ and has a non-zero residue $K$ at $s=n$. Let $C=\operatorname{Tr}_{R}\left(\mathscr{D}^{-n}\right)$. Then we note that $K$ is of the form $K=C \frac{\chi_{\pi}(e)}{|\Gamma|}$. The asymptotic estimate follows by direct application of a Tauberian theorem (See for example [14], Theorem 4.1).

Let us now consider a pair of irreducible representations $\pi_{j}, j=1,2$, of equal dimension and compare their occurrence in eigenspaces of our invariant operator $\mathscr{D}$. We shall assume that for all but finitely many eigenvalues $\lambda_{i}$,

$$
\left\langle V_{\lambda_{i}}, \pi_{1}\right\rangle \geq\left\langle V_{\lambda_{i}}, \pi_{1}\right\rangle .
$$

Applying the same argument as above we obtain the following.

Corollary 3.3. Suppose that $\pi_{j}$ are irreducible representations as above and let $k=\max _{g \in \Gamma}\left\{\operatorname{dim} M^{g} \mid \chi_{\pi_{1}}(g) \neq \chi_{\pi_{2}}(g)\right\}$. Then asymptotically,

$$
N_{\pi_{1}, \pi_{2}, \mathscr{D}}(\lambda):=\sum_{\lambda_{i} \leq \lambda}\left\langle V_{\lambda_{i}}, \pi_{1}-\pi_{2}\right\rangle \simeq C \lambda^{k} .
$$

\section{Hochschild and cyclic homology for cross-products}

The Hochschild and cyclic homology of algebras play the role of differential forms and de Rham cohomology in Connes' noncommutative geometry [9]. Our main goal is to compute the homologies for the cross-product algebra $\mathcal{A}(M) \rtimes \Gamma$. In this section we shall begin with a mundane approach to compute homologies for cross-product algebras.

Let $\mathcal{A}$ be an algebra and $\Gamma$ be a finite group acting on it by $\pi: \Gamma \rightarrow \operatorname{Aut}(\mathcal{A})$. For most purposes, $\mathcal{A}$ will be a unital locally convex topological algebra with jointly 
continuous product. We define the cross-product algebra $\mathcal{B}=\mathcal{A} \rtimes \Gamma$ as the algebra generated by elements of the form $\left\{a_{g} g \mid a_{g} \in \mathcal{A}, g \in \Gamma\right\}$ with the product given by

$$
a_{g} g \cdot b_{h} h=a_{g} g\left(b_{h}\right) g h .
$$

The Hochschild homology of $\mathscr{B}$ admits a natural decomposition. For every conjugacy class $\langle\gamma\rangle$ of the group $\Gamma$, there is a subcomplex of $\mathscr{H}_{*}(\mathscr{B})$ given by

$$
\mathscr{H}_{*}(\mathcal{A})_{\gamma}=\left\{\left(a_{g_{0}} g_{0} \otimes a_{g_{1}} g_{1} \otimes \cdots \otimes a_{g_{n}} g_{n}\right) \mid g_{1} \cdot g_{2} \cdots g_{n} \cdot g_{0} \in\langle\gamma\rangle\right\},
$$

which yields the decomposition

$$
\mathscr{H}_{*}(\mathscr{B})=\bigoplus_{\langle\gamma\rangle} \mathscr{H}_{*}(\mathcal{A})_{\gamma} .
$$

Here we shall also use the notation $L_{*}(\mathscr{A}, \Gamma, \gamma)$ for $\mathscr{H}_{*}(\mathcal{A})_{\gamma}$ if we need to specify the group $\Gamma$ explicitly.

Let us first identify the homologies of each of the conjugacy component $\mathscr{H}_{*}(\mathcal{A})_{\gamma}$ with that of a certain twisted Hochschild complex.

4.1. Twisted Hochschild complex for commutative algebras. We consider a commutative algebra $\mathcal{A}$. Let us as usual write $\mathcal{A}^{e}=\mathcal{A} \otimes \mathcal{A}$. Given an automorphism $h$ of $\mathcal{A}$, consider the $\mathcal{A}^{e}$ module $\mathcal{A}_{h}$ with the same linear structure as $\mathcal{A}$, but the module structure given by

$$
(a \otimes b) \cdot c=a c \cdot h(b) .
$$

Furthermore let us consider the complex $\mathcal{C}_{*}(\mathcal{A})_{h}=\bigoplus \mathcal{A}^{\otimes n+1}, b_{h}$, where the twisted Hochschild differential $b_{h}$ is defined by

$$
\begin{aligned}
b_{h}\left(a_{0} \otimes a_{1} \otimes \cdots \otimes a_{n}\right)= & \left(a_{0} h\left(a_{1}\right) \otimes \cdots \otimes a_{n}\right) \\
& +\sum_{i=1}^{n}(-1)^{i}\left(a_{0} \otimes \cdots \otimes a_{i} a_{i+1} \otimes \cdots \otimes a_{n}\right) .
\end{aligned}
$$

In case of a group action on a manifold $M$ we shall be able to very easily identify the homology of the twisted complex $\mathcal{C}_{*}\left(\mathcal{C}^{\infty}(M)\right)_{h}$ with the differential forms on the fixed point manifold $M^{h}$. Hence in this section we show that twisted homology gives the same homology as the conjugacy component $\mathscr{H}_{*}\left(\mathcal{C}^{\infty}(M) \rtimes \Gamma\right)_{\langle h\rangle}$.

To begin with let us make the following observation.

Lemma 4.1. We have

$$
\mathscr{H}\left(\mathcal{C}_{*}(\mathcal{A})_{h}, b_{h}\right) \simeq \operatorname{Tor}_{*}^{\mathcal{A}^{e}}\left(\mathcal{A}, \mathcal{A}_{h}\right) .
$$

Proof. This is a simple direct calculation. Let us introduce $\mathscr{H}_{*}^{\prime}(\mathcal{A})$ to be the bar resolution for a unital algebra $\mathcal{A}$. That is, $\mathscr{H}_{*}^{\prime}(\mathcal{A})=\left(\mathcal{A}^{\otimes n+1}, b^{\prime}\right)$ is the standard projective resolution of $\mathcal{A}$ by $\mathcal{A}^{e}$ modules. Consider the map

$$
\begin{gathered}
\psi: \mathscr{H}_{n}(\mathcal{A}) \otimes_{\mathcal{A}^{e}} \mathcal{A}_{h} \rightarrow \mathcal{C}_{n}(\mathcal{A})_{h}, \\
\psi\left(a_{0} \otimes a_{1} \otimes \cdots \otimes a_{n}\right) \otimes a \rightarrow\left(a_{n} a h\left(a_{0}\right) \otimes a_{1} \otimes \cdots \otimes a_{n-1}\right) .
\end{gathered}
$$


Then we check that $b_{h} \circ \psi=\psi \circ b^{\prime} \otimes 1$, which means that $\psi$ is a morphism of complexes. The result follows from the fact that $\psi$ is an isomorphism and the definition of the Tor groups.

Abstractly the above result tells us that the twisted Hochschild complex $\mathscr{C}_{*}(\mathcal{A})_{h}$ has the same homology as the conjugacy component $\mathscr{H}_{*}(\mathcal{A})_{\langle h\rangle}$, but we are looking for a concrete quasi-isomorphism.

To this end we begin with another well-known acyclic model. For any finite group $G$, let $\beta G:=(\beta G)_{n}=\mathbb{C}[\underbrace{G \times G \times \cdots \times G}_{n \text { times }}]$ be the complex endowed with the differential

$$
\bar{d}\left(g_{0}, g_{1}, \ldots, g_{n}\right)=\sum_{i=0}^{n}(-1)^{i}\left(g_{0}, g_{1}, \ldots, \widehat{g_{i}}, \ldots, g_{n}\right),
$$

where the notation $\widehat{g_{i}}$ as usual means that the entry is omitted. This differential also comes from a simplicial object structure on $\beta G$. It is well known that

$$
H_{q}(\beta G)= \begin{cases}\mathbb{C} & \text { if } q=0, \\ 0 & \text { if } q>0,\end{cases}
$$

and hence $\beta G$ is a free resolution for the trivial $G$ module $\mathbb{C}$.

For any subgroup $G \subseteq \Gamma$, we define the complex

$$
\tilde{L}_{n}(\mathcal{A}, G, h):=\left(\mathcal{C}_{*}(\mathcal{A})_{h}\right)_{n} \otimes \beta G_{n}
$$

with induced simplicial structure and differential given by

$$
\begin{aligned}
b_{h} \otimes \bar{d}\left(a_{0} g_{0}, a_{1} g_{1}, \ldots, a_{n} g_{n}\right)= & \left(a_{0} h\left(a_{1}\right) g_{1}, a_{2} g_{2}, \ldots, a_{n} g_{n}\right) \\
& +\sum_{i=1}^{n-1}(-1)^{i}\left(a_{0} g_{0}, \ldots, a_{i} a_{i+1} g_{i+1}, \ldots, a_{n} g_{n}\right) \\
& +(-1)^{n}\left(a_{n} a_{0} g_{0}, a_{1} g_{1} \ldots, a_{n-1} g_{n-1}\right) .
\end{aligned}
$$

As one would expect this $\tilde{L}_{*}(\mathcal{A}, G, h)$ complex is a simply connected cover of $\mathscr{H}_{*}(\mathcal{A})_{\langle h\rangle}$. ( See [23] for a more detailed presentation.)

An application of the Eilenberg-Zilber isomorphism (see [20], Ch. VIII.8) gives us the following result. Throughout we shall keep track of our quasi-isomorphisms.

Lemma 4.2. $\tilde{L}(\mathcal{A}, G, h)$ is quasi-isomorphic to $\mathcal{C}_{*}(\mathcal{A})_{h}$.

Proof. By definition, $\tilde{L}(\mathcal{A}, G, h)_{n} \simeq \beta G_{n} \otimes\left(\mathcal{C}_{*}(\mathcal{A})_{h}\right)_{n}$. Thus by the Künneth formula and the Eilenberg-Zilber theorem, we have the diagram

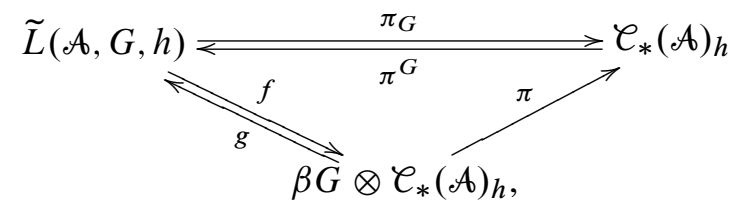


where we write

$$
\pi_{G}\left(a_{0} g_{0}, a_{1} g_{1}, \ldots, a_{n} g_{n}\right):=\left(a_{0}, a_{1}, \ldots, a_{n}\right)
$$

and similarly

$$
\pi^{G}\left(a_{0}, a_{1}, \ldots, a_{n}\right):=\left(a_{0} e, a_{1} e, \ldots, a_{n} e\right) .
$$

The maps $f$ and $g$ are the maps for the Eilenberg-Zilber quasi-isomorphism, and $\pi$ is the projection on the first component. (The Eilenberg-Zilber isomorphism applies because the complex $\widetilde{L}(\mathcal{A}, G, h)$ is obtained from the product of two simplicial objects.) That is,

$$
\pi\left((\beta G)_{l} \otimes \mathscr{C}_{k}(\mathcal{A})\right)= \begin{cases}0 & \text { if } l \neq 0 \\ \mathscr{C}_{k}(\mathcal{A}) & \text { if } l=0 .\end{cases}
$$

Since $H_{q}(\beta G)=0$ for $q>0, \pi$ turns out to be a quasi-isomorphism. Thus $\pi_{G}$ and $\pi^{G}$ are quasi-isomorphisms.

Let $\mathcal{A}$ be a commutative unital algebra with an action of a group $\Gamma$. Let $h \in\langle\gamma\rangle$ be an element of the conjugacy class $\langle\gamma\rangle$ and $\mathcal{C}_{*}(A)_{h}$ be the corresponding twisted complex under the action of $h$, then we can make the following identifications:

Proposition 4.3. The conjugacy component of the Hochschild homology $\mathscr{H}_{*}(\mathcal{A})_{h}$ is quasi-isomorphic to $\mathcal{C}_{*}(\mathcal{A})_{h} \Gamma_{h}$.

The chain map $G: \mathscr{H}_{*}(\mathcal{A})\langle\gamma\rangle \rightarrow \mathcal{C}_{*}(\mathcal{A})_{h}^{\Gamma_{h}}$ is given by the explicit formula

$$
\begin{aligned}
& G_{h}\left(b_{0} h_{0}, b_{1} h_{1}, \ldots, b_{n} h_{n}\right) \\
& \quad=\frac{1}{\left|\Gamma_{h}\right|} \sum_{g_{0} \in \Gamma_{h}}\left(h g_{0} h_{0}^{-1}\left(b_{0}\right), g_{0}\left(b_{1}\right), \ldots, g_{0} h_{1} \ldots h_{n-1}\left(b_{n}\right)\right) .
\end{aligned}
$$

Proof. There is a covering map $\alpha: \widetilde{L}(\mathcal{A}, \Gamma, h) \rightarrow \mathscr{H}_{*}(\mathcal{A})_{\gamma}$ given by

$$
\begin{aligned}
\alpha\left(a_{0} g_{0},\right. & \left.a_{1} g_{1}, \ldots, a_{n} g_{n}\right) \\
& =\left(g_{n}^{-1} a_{0} h g_{0}, g_{0}^{-1} a_{1} g_{1}, \ldots, g_{n-1}^{-1} a_{n} g_{n}\right) \\
\quad= & \left(g_{n}^{-1}\left(a_{0}\right) g_{n}^{-1} h g_{0}, g_{0}^{-1}\left(a_{1}\right) g_{0}^{-1} g_{1}, \ldots, g_{n-1}^{-1}\left(a_{n}\right) g_{n-1}^{-1} g_{n}\right),
\end{aligned}
$$

which is a chain map. In fact, $\alpha$ is a morphism of simplicial objects and $\Gamma_{h}$ equivariant. We would like to lift this map $\alpha$ from $\mathscr{H}_{*}(\mathcal{A})_{\langle h\rangle}$ to $\widetilde{L}(\mathcal{A}, \Gamma, h)^{\Gamma_{h}}$.

The map $\alpha$ above also restricts to a chain map on the quasi-isomorphic complex $\tilde{L}\left(\mathcal{A}, \Gamma_{h}, h\right)$ which we denote by $\left.\alpha\right|_{\Gamma_{h}}$. An explicit lifting is easy to construct for $\alpha_{\Gamma_{h}}$ as follows: 
Define, for any $g_{0} \in \Gamma_{h}$ a linear map $T_{g_{0}}: L\left(\mathcal{A}, \Gamma_{h}, h\right) \rightarrow \widetilde{L}\left(\mathcal{A}, \Gamma_{h}, h\right)$ by the formula

$$
\begin{aligned}
T_{g_{0}}\left(b_{0} h_{0}, b_{1} h_{1}, \ldots, b_{n} h_{n}\right)= & \left(h g_{0} h_{0}^{-1}\left(b_{0}\right) g_{0}, g_{0}\left(b_{1}\right) g_{0} h_{1}, \ldots\right. \\
& \ldots, g_{0} h_{1} \ldots h_{i-1}\left(b_{i-1}\right) g_{0} h_{1} \ldots h_{i}, \ldots \\
& \left.\ldots, g_{0} h_{1} \ldots h_{n-1}\left(b_{n}\right) g_{0} h_{1} \ldots h_{n}\right) .
\end{aligned}
$$

Then verify directly that

$$
T_{g_{0}} b_{h}-b T_{g_{0}}=0 .
$$

Let us define the map $T=\frac{1}{\left|\Gamma_{h}\right|} \sum_{g_{0} \in \Gamma_{h}} T_{g_{0}}$. Clearly $T$ maps $L\left(\mathcal{A} . \Gamma_{h}, h\right)$ to $\tilde{L}\left(\mathcal{A}, \Gamma_{h}, h\right)^{\Gamma_{h}}$. We next observe that $\alpha \circ T=\operatorname{Id}_{\mathscr{H}_{*}(\mathcal{A})_{\gamma}}$ and $T \circ \alpha=\operatorname{Id}_{\tilde{L}(\mathcal{A}, \Gamma, h)^{\Gamma}}$ and that $T=\alpha^{-1}$ and therefore must be a chain map. Thus we have established the following commutative diagram of quasi-isomorphisms:

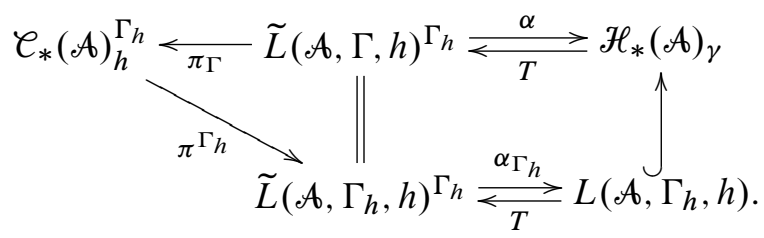

Here $F:=\alpha \circ \pi^{\Gamma}: \mathcal{C}_{*}(\mathcal{A})_{h}^{\Gamma_{h}} \rightarrow \mathscr{H}_{*}(\mathcal{A})_{\gamma}$ is of the form

$$
F\left(a_{0}, a_{1}, \ldots, a_{n}\right)=\left(a_{0} h, a_{1} e, \ldots a_{n} e\right) .
$$

At the same time the inverse quasi-isomorphism $G:=\pi_{\Gamma} \circ T: \mathscr{H}_{*}(\mathcal{A})_{\langle\gamma\rangle} \rightarrow \mathcal{C}_{*}(\mathcal{A})_{h}$ is given by the formula (3)

Thus as a consequence we immediately have that

$$
\mathscr{H}_{*}(\mathcal{A})_{\langle\gamma\rangle} \simeq \mathcal{C}_{*}(\mathcal{A})_{h}^{\Gamma_{h}} \simeq \operatorname{Tor}_{*}^{A^{e}}\left(\mathcal{A}, \mathcal{A}_{h}\right)^{\Gamma_{h}} .
$$

As already mentioned earlier the computation shall be of interest in case of $\mathcal{A}=\mathcal{C}^{\infty}(M)$. We now begin our efforts to show that in this case homology of $C_{*}\left(C^{\infty}(M)\right)_{h}$ can be described by differential forms on the fixed point sets $M^{h}$ with explicit identification given using the formula (3).

4.2. Local computations. Let us first specialize to the case when the algebra $\mathcal{A}=$ $e^{\infty}(V)$ is the algebra of smooth functions on a vector space $V$ and $\gamma: V \rightarrow V$ is a linear transformation. In this section we shall identify our twisted Hochschild homology that is the homology of the complex $\mathscr{C}_{*}(\mathcal{A})_{\gamma}$ with the differential forms on the fixed point subspace $V^{\gamma}$. To this end it is most convenient to introduce the language of Koszul complexes. 
4.3. Koszul complex. Let $R$ be a commutative ring. Let $f_{1}, f_{2}, \ldots, f_{q} \in R$. Let $\left\{v_{j}\right\}$ be a basis for $\mathbb{C}^{q}$. We define the Koszul complex of $R$ generated by $f_{1}, f_{2}, \ldots$, $f_{q}$ by

$$
\begin{gathered}
\mathcal{K}_{l}\left(R: f_{1}, f_{2}, \ldots, f_{q}\right)=R \otimes \wedge^{l} \mathbb{C}^{q}, \\
\delta\left(r \otimes v_{i_{1}} \wedge v_{i_{2}} \wedge \cdots \wedge v_{i_{l}}\right)=\sum_{j=1}^{j=q}(-1)^{j}\left(r f_{j} \otimes v_{i_{1}} \wedge v_{i_{2}} \cdots \wedge \widehat{v_{i_{j}}} \wedge \cdots \wedge v_{i_{l}}\right) .
\end{gathered}
$$

This differential arises naturally from a simplicial module structure. We observe the following properties:

Lemma 4.4. (1) Let $R, R^{\prime}$ be two algebras over $\mathbb{C}$ and let $S \subset R$ and $S^{\prime} \subset R^{\prime}$ be subsets. Denote by $S \amalg S^{\prime}=S \otimes 1 \cup 1 \otimes S^{\prime} \subset R \otimes R^{\prime}$. Then

$$
\mathcal{K}_{*}\left(R \otimes R^{\prime}: S \amalg S^{\prime}\right)=\mathcal{K}_{*}(R: S) \otimes \mathcal{K}_{*}\left(R^{\prime}: S^{\prime}\right) .
$$

(2) Let $V=\mathbb{R}^{n}$. Let $\mathcal{A}=C^{\infty}(V)$, and let $X_{i}$ be the coordinate functions on $V$. Then

$$
\mathscr{H}_{q}\left(\mathcal{K}_{*}\left(\mathcal{A}:\left\{X_{i}\right\}_{i=1}^{n}\right)= \begin{cases}0 & \text { if } q>0, \\ \mathbb{C} & \text { if } q=0 .\end{cases}\right.
$$

Proof. Since the differential comes from a simplicial object structure the first fact is a consequence of the Eilenberg-Zilber theorem.

The second fact follows from the Poincare lemma and properties of the Fourier transform.

Given a linear transformation $\gamma$ of a real vector space $V$ we decompose $V$ into a fixed point subspace $V^{\gamma}$ and an invariant complement $(1-\gamma) V$,

$$
V=V^{\gamma} \oplus(1-\gamma) V .
$$

We come now to the main result of this section which is the local version of our desired result.

Theorem 4.5. Let $\gamma$ be a linear automorphism of the algebra $\mathcal{A}=\bigodot^{\infty}(V)$. The homology of the twisted complex $\left(C_{*}(A)_{\gamma}, b_{\gamma}\right)$ is then given by the space of forms on the fixed point $V^{\gamma}$,

$$
H_{q}\left(C_{*}(A)_{\gamma}\right) \simeq \Omega^{q}\left(V^{\gamma}\right),
$$

and the identification is $\Gamma$ equivariant.

Proof. Let $e_{i}$ be a basis of $V$ such that $e_{i} \in V^{\gamma}$ for $1 \leq i \leq m$ and $e_{i} \in(1-\gamma) V$ for $m+1 \leq i \leq n$. Let $X_{i}$ denote the corresponding coordinate functions.

Consider the Koszul complex

$$
\mathcal{K}_{*}\left(\mathcal{A}^{e}:\left\{X_{i} \otimes 1-1 \otimes X_{i}\right\}_{i=1}^{n}\right),
$$


which is a projective resolution of $\mathcal{A}$ over $\mathcal{A}^{e}$. Hence by Lemma 4.1,

$$
\boldsymbol{C}_{*}(\mathcal{A})_{\gamma} \simeq \mathcal{K}_{*}\left(\mathcal{A}^{e}:\left\{X_{i} \otimes 1-1 \otimes X_{i}\right\}_{i=1}^{n}\right) \otimes_{\mathcal{A}^{e}} \mathcal{A}_{\gamma} .
$$

Observe that $\mathcal{K}_{*}\left(\mathcal{A}^{e}:\left\{X_{i} \otimes 1-1 \otimes X_{i}\right\}_{i=1}^{n}\right) \otimes_{\mathcal{A}^{e}} \mathcal{A}_{\gamma}$ can be identified with another Koszul complex, namely $\mathcal{K}_{*}\left(\mathcal{A}:\left\{X_{i}-\gamma\left(X_{i}\right)\right\}\right)$ via the map

$$
\psi\left(a \otimes b \otimes v_{i_{1}} \wedge \cdots \wedge v_{i_{l}} \otimes c\right)=a \gamma(b) c \otimes v_{i_{1}} \wedge \cdots \wedge v_{i_{l}} .
$$

This results in the diagram

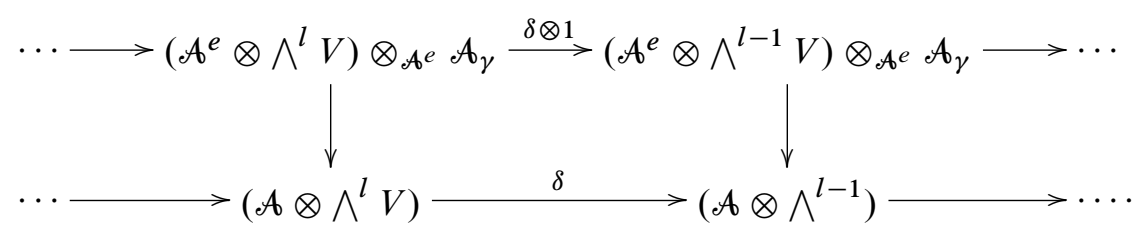

Let $R=C^{\infty}\left(V^{\gamma}\right)$ be smooth functions on the fixed point manifold $V^{\gamma}$ and let $R^{\prime}=C^{\infty}((1-\gamma) V)$ be smooth functions on the invariant complement of $V^{\gamma}$.

Since $\mathcal{A}=C^{\infty}(V)=C^{\infty}\left(V^{\gamma}\right) \otimes C^{\infty}((1-\gamma) V)=R \otimes R^{\prime}$, by Lemma 4.4 (1),

$$
\begin{aligned}
\mathcal{K}(\mathcal{A}: & \left.\left\{X_{i}-\gamma\left(X_{i}\right)\right\}_{i=1}^{n+q}\right) \\
& =\mathcal{K}\left(R:\left\{X_{i}-\gamma\left(X_{i}\right)\right\}_{i=1}^{m}\right) \otimes \mathcal{K}\left(R^{\prime}:\left\{X_{i}-\gamma\left(X_{i}\right)\right\}_{i=m+1}^{n+q}\right) \\
& \simeq \mathcal{K}(R:\{0\}) \otimes \mathcal{K}\left(R^{\prime}:\left\{X_{i}\right\}_{i=m+1}^{m+q}\right) .
\end{aligned}
$$

Now by applying Lemma $4.4(2)$ to $\mathcal{K}\left(R^{\prime}:\left\{X_{i}\right\}_{i=m+1}^{m+q}\right)$, we have

$$
\mathcal{K}\left(\mathcal{A}:\left\{X_{i}-\gamma\left(X_{i}\right)\right\}_{i=1}^{n+q}\right)=\mathcal{K}(R:\{0\}) \simeq \Omega\left(V^{\gamma}\right) .
$$

Here the last identification is due to the following theorem, which we formulate only in the $\sigma$-compact case, for simplicity.

Theorem 4.6 (Connes' HKR Theorem). Let $X$ be a smooth, $\sigma$-compact manifold. Then the Hochschild homology of the algebra $\mathcal{A}=\bigodot^{\infty}(X)$ is given by the differential forms on $X$. The map

$$
\chi_{k}\left(a_{0} \otimes a_{1} \otimes \cdots \otimes a_{k}\right) \rightarrow a_{0} d a_{1} d a_{2} \ldots d a_{k}
$$

induces an isomorphism

$$
\mathrm{HH}_{k}\left(e^{\infty}(X)=\Omega^{k}(X) .\right.
$$

Proof. Let us consider $X=\mathbb{R}^{n}$, which is sufficient to prove the equality in (4). It is easily seen that the map $\chi$ is a chain map. We define the inverse map (which is only well defined on the homology)

$$
\begin{gathered}
E_{*}: \Omega^{*}\left(\mathbb{R}^{n}\right) \rightarrow \mathscr{H}_{*}\left(\mathcal{C}^{\infty}\left(\mathbb{R}^{n}\right)\right), \\
E_{k}\left(a_{0} d a_{1} d a_{2} \ldots d a_{k}\right)=\frac{1}{k !} \sum_{\pi \in S_{k}} \operatorname{sign}(\pi)\left(a_{0} \otimes a_{\pi(1)} \otimes a_{\pi(2)} \otimes \cdots \otimes a_{\pi(k)}\right) .
\end{gathered}
$$


Then the following diagram commutes.

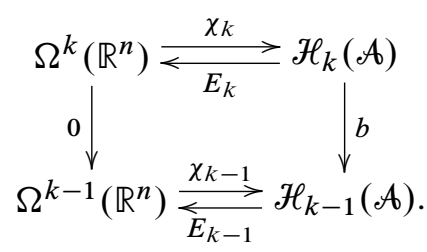

Then the map $\chi_{k}$ induces an isomorphism

$$
\mathrm{HH}_{k}\left(\mathcal{C}^{\infty}\left(\mathbb{R}^{n}\right)\right) \simeq \Omega^{k}\left(\mathbb{R}^{n}\right) .
$$

The case of a closed manifold $X$ will be proved in the next section.

Thus combining all this information we get the following diagram.

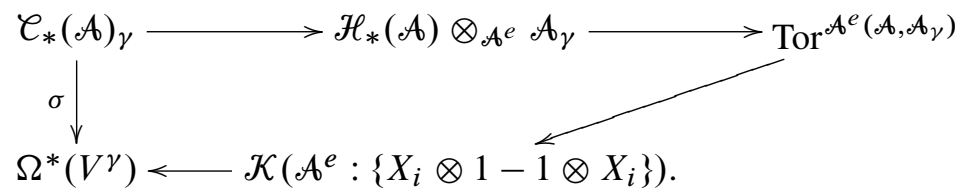

We note that all the maps involved are $\Gamma$ equivariant.

The next result now follows immediately by Proposition 4.3 .

Corollary 4.7. Let $\Gamma$ be a finite group acting linearly on $\mathcal{A}=\mathcal{C}^{\infty}(V)$ and $\mathscr{B}=\mathcal{A} \rtimes \Gamma$ and let $\gamma \in \Gamma$. Then the $\langle\gamma\rangle$ component of the Hochschild homology $\mathrm{HH}(\mathscr{B})_{\gamma}$ is given by

$$
\mathrm{HH}_{*}(\mathcal{B})_{\gamma}=\Omega\left(V^{\gamma}\right)^{\Gamma_{\gamma}} .
$$

\section{Equivariant HKR theorem}

Thus far we have prepared for computing the Hochschild and cyclic homology of $\bigodot^{\infty}(M) \rtimes \Gamma$. In order to do so it suffices to compute the homology $\ell_{*}\left(\complement^{\infty}(M)\right)_{h}$ where $h \in \Gamma$ varies over a set of representatives of conjugacy classes.

Proposition 5.1. The map $\tilde{\chi}_{*}: \mathcal{C}_{*}\left(\mathcal{C}^{\infty}(M)\right)_{h} \rightarrow \Omega^{*}\left(M^{h}\right), \tilde{\chi}_{n}\left(a_{0} \otimes a_{1} \otimes \ldots a_{n}\right):=$ $\left.a_{0} d a_{1} d a_{2} \ldots d a_{n}\right|_{M^{h}}$, is a quasi-isomorphism.

Proof. We shall identify the complex $\mathcal{C}_{*}\left(\mathcal{C}^{\infty}(M)\right)_{h}$ with a complex of sheaves over $M^{h}$ as follows. First we shall identify $\bigodot^{\infty}(M)^{\otimes n+1}$ with $\bigodot^{\infty}\left(M^{n+1}\right)$. With this 
identification made the differential $b_{h}$ takes the following form:

$$
\begin{aligned}
b_{h} F\left(x_{0}, x_{1}, \ldots, x_{n-1}\right)= & F\left(x_{0}, h^{-1} x_{0}, x_{1}, \ldots, x_{n-1}\right) \\
& +\sum_{i=1}^{n-2}(-1)^{i} F\left(x_{0}, x_{1}, \ldots x_{i}, x_{i}, \ldots, x_{n-1}\right) \\
& +(-1)^{n-1} F\left(x_{0}, x_{1}, \ldots, x_{n-1}, x_{0}\right) .
\end{aligned}
$$

Let $i_{n}: M^{h} \rightarrow M^{n+1}$ be the inclusion of $M^{h}$ in $M^{n+1}$ given by $s \rightarrow(x, x, \ldots x)$. We shall denote by $\Delta_{h}^{n}$ the image under this embedding. We also define

$$
J_{n}:=\left\{f \in \mathcal{C}^{\infty}\left(M^{n+1}\right) \mid \operatorname{support}(f) \operatorname{disjoint} \text { from } \Delta_{h}^{n}\right\} .
$$

We shall prove at the end of this section that the following normalization result holds.

Lemma 5.2. The complex $\left(J_{n}, b_{h}\right)$ is an acyclic subcomplex of $\mathcal{C}_{n}\left(\mathcal{C}^{\infty}(M)\right)_{h}$ and hence the quotient map $\left.\ell_{*}\left(\mathcal{C}^{\infty}(M)\right)_{h}\right) \rightarrow \mathcal{C}_{*}\left(\mathcal{C}^{\infty}(M)\right)_{h} / J_{*}$ is a quasi-isomorphism.

Granting this result for the moment it follows immediately that if $\mathfrak{C}(X)$ denotes the sheaf of smooth functions on a manifold $X$, then $\mathcal{K}_{n}:=i_{n}^{-1} \mathfrak{C}\left(M^{n+1}\right)$ and $b_{h}$ forms a complex of sheaves on $M^{h}$ with the property

$$
\mathscr{H}_{*}\left(\mathcal{C}_{*}\left(\mathcal{C}^{\infty}(M)\right)_{h}\right) \simeq \mathscr{H}_{*}\left(\Gamma\left(M^{h}, \mathcal{K}_{*}\right) .\right.
$$

Furthermore, the map $\tilde{\chi}_{*}: \mathcal{C}_{*}\left(\mathcal{C}^{\infty}(M)\right)_{h} \rightarrow \Omega^{*}\left(M^{h}\right)$ factors through the quotient $\Gamma\left(M^{h}, \mathcal{K}_{*}\right)$ and determines a sheaf morphism still called $\chi_{*}$. Theorem 4.5 is precisely the statement that $\chi_{*}$ induces an isomorphism at the level of stalks providing

$$
H_{*}\left(\mathcal{K}_{*, p}\right) \simeq \Omega_{p}^{*}\left(M^{h}\right)
$$

at each point $p \in M^{h}$. Since $\mathcal{K}_{n}$ are flabby sheaves, the homology and section functor commute and we obtain that $\chi_{*}$ is a quasi-isomorphism as desired.

The result on computing the homology of the cross-product algebra $\bigodot^{\infty}(M) \rtimes \Gamma$ now follows immediately from Proposition 5.1 and (3).

Theorem 5.3. The map

$$
\chi_{*}^{h}:=\tilde{\chi}_{*} \cdot G_{h}: \mathscr{H}_{*}\left(e^{\infty}(M) \rtimes \Gamma\right)_{\langle\gamma\rangle} \rightarrow\left(\Omega^{*}\left(M^{h}\right)\right)^{\Gamma_{h}}
$$

is a quasi-isomorphism. Explicitly,

$$
\begin{aligned}
& \chi_{n}^{h}\left(a_{0} g_{0}, a_{1} g_{1}, \ldots, a_{n} g_{n}\right) \\
& \quad=\left.\frac{1}{\left|\Gamma_{h}\right|} \sum_{\alpha \in \Gamma_{h}}\left(h \alpha g_{0}^{-1}\left(a_{0}\right) d \alpha\left(a_{1}\right), \ldots d \alpha g_{1} \ldots g_{n-1}\left(a_{n}\right)\right)\right|_{M^{h}} .
\end{aligned}
$$


We note here also that from the proof of Theorem 4.5 it follows that the inverse (defined only on homology) is given by

$E_{k}\left(a_{0} d a_{1} d a_{2} \ldots d a_{k}\right)=\frac{1}{k !} \sum_{\pi \in S_{k}} \operatorname{sign}(\pi)\left(\tilde{a}_{0} \gamma \otimes \tilde{a}_{\pi(1)} e \otimes \tilde{a}_{\pi(2)} e \otimes \cdots \otimes \tilde{a}_{\pi(k)} e\right)$,

where each $\tilde{a}_{j}$ is an extension of the function $a_{j}$ on $M_{h}$ which is $\Gamma_{h}$ invariant and is obtained by identifying a tubular neighbourhood of $M^{h}$ with $(1-g) T M^{h}$.

The following computations of cyclic and periodic cyclic homology are routine.

Theorem 5.4. Let $\langle\gamma\rangle$ be a conjugacy class of $\Gamma$ and $h \in\langle\gamma\rangle$.

$$
\mathrm{HC}_{k}\left(\mathcal{C}^{\infty}(M) \rtimes \Gamma\right)_{\gamma}=\Omega^{k}\left(M^{\gamma}\right)^{\Gamma_{\gamma}} \oplus \underset{j>0}{\bigoplus} H_{\mathrm{deRham}}^{k-2 j}\left(M^{\gamma}\right)^{\Gamma_{\gamma}} .
$$

Proof. Since $\chi:\left(B_{*}\left(\complement^{\infty}(M) \rtimes \Gamma\right)_{\gamma}, b, B\right) \rightarrow\left(\Omega^{*}\left(M^{\gamma}\right)^{\Gamma_{\gamma}}, 0, d\right)$ is a map of mixed complexes which is an isomorphism on the columns by Theorem 5.3 above, $\chi^{\gamma}$ must be an isomorphism on the total complexes of these mixed complexes.

Corollary 5.5. We have

$$
\operatorname{HP}_{k}(\mathscr{B})_{\gamma}=\sum_{j \in \mathbb{Z}} H_{\mathrm{deRham}}^{k-2 j}\left(M^{\gamma}\right)^{\Gamma_{\gamma}}
$$

Proof. In view of $\mathrm{HH}_{n}(\mathscr{B})_{\gamma}=0$ for $n>\operatorname{dim} M$, the periodicity map $S: \mathrm{HC}_{n+2}(\mathscr{B})_{\gamma} \rightarrow \mathrm{HC}_{n}(\mathscr{B})_{\gamma}$ is an isomorphism by the SBI-exact sequence. In particular, $\lim _{\longleftarrow} \mathrm{HC}_{*}(\mathscr{B})_{\gamma}=\sum_{j \in \mathbb{Z}} H_{\text {deRham }}^{k-2 j}\left(M^{\gamma}\right)^{\Gamma_{\gamma}}$. Also $\lim ^{1} \mathrm{CC}_{*}(\mathscr{B})_{\gamma}=0$.

We complete this discussion with a proof of the normalization result in Theorem 5.1.

Proof of Lemma 5.2. This proof is analogous to the resolution of the normalized complex for a simplicial object. There is a filtration of the subcomplex $J_{n}$ constructed as follows.

Let $T_{i}: M \rightarrow M$ be the identity map when $i \neq 0$ and $T_{0}=h^{-1}$. We consider open subsets $\mathcal{V}_{j}^{n+1} \subset M^{n+1}$ for $j=0,1, \ldots, n$ defined by

$$
\left(x_{0}, x_{1}, \ldots, x_{n}\right) \in \mathcal{V}_{j}^{n+1} \Longleftrightarrow x_{j+1} \neq T_{j} x_{j}
$$

(Here we use the convention $n+1=0$.) The necessary filtration is then described by

$$
F_{i} J_{n}=\sum_{j=0}^{i}\left\{f \in \mathcal { C } ^ { \infty } \left(M^{n+1} \mid f \in \mathcal{C}_{c}^{\infty}\left(V_{j}^{n+1}\right\} .\right.\right.
$$

The differential induced on the corresponding associated graded algebra is easily seen to be zero. 


\section{Topologically filtered algebras}

The algebra of complete symbols with the topology that is described below is not a topological algebra in the sense that multiplication in $\mathcal{A}(M)$ is not jointly continuous. Hence we need a larger category, namely that of topologically filtered algebras defined in [4]. We present a simplified category here. Let $\mathcal{A}$ be an algebra with filtration $\mathcal{A}=\bigcup_{p \in \mathbb{Z}} \mathcal{F}_{p} \mathcal{A}$. That is to say, each $\mathcal{F}_{p} \mathcal{A}$ is a subspace $\mathcal{F}_{p} \mathcal{A} \subset \mathcal{F}_{p+1} \mathcal{A}$ and the multiplication map takes $\mathscr{F}_{p} \mathcal{A} \times \mathscr{F}_{q} \mathcal{A} \rightarrow \mathscr{F}_{p+q} \mathcal{A}$. We will say that $\mathcal{A}$ is a filtered algebra for short.

Since, by definition, $\mathcal{F}_{0} \mathcal{A}$ is a subalgebra of $\mathcal{A}$ and $\mathcal{F}_{-j} \mathcal{A}$ is an ideal of $\mathcal{F}_{0} \mathcal{A}$, $\mathcal{M}_{0}^{j}:=\mathcal{F}_{0} \mathcal{A} / \widetilde{F}_{-j} \mathcal{A}$ is naturally an algebra and each $\mathcal{M}_{p}^{j}:=\mathcal{F}_{p} \mathcal{A} / \mathcal{F}_{p-j} \mathcal{A}$ is a module over $\mathcal{M}_{0}^{j}$. Similarly, $\mathcal{I}:=\bigcap_{p} \mathcal{F}_{p} \mathcal{A}$ is an ideal in $\mathcal{A}$. We call our algebra a symbol algebra if $\mathcal{I}=0$. We only consider symbol algebras for now. That is, if $\mathcal{A}^{\prime}$ is any filtered algebra, we consider the algebra $\mathcal{A}=\mathcal{A}^{\prime} / \mathcal{I}$.

Definition 6.1. We say that a filtered algebra is topologically filtered if:

(1) $\mathcal{A}$ is a symbol algebra, that is, $\mathcal{I}:=\bigcap_{p} \mathcal{F}_{p} \mathcal{A}=0$.

(2) Each $\mathcal{M}_{p}^{j}=\mathscr{F}_{p} \mathcal{A} / \mathscr{F}_{p-j} \mathcal{A}$ is a nuclear Fréchet space for all $p$ and each $j$.

(3) Each module map $\mathcal{M}_{p}^{j} \widehat{\otimes} \mathcal{M}_{q}^{j} \rightarrow \mathcal{M}_{p+q}^{j}$ induced by the multiplication in $\mathcal{A}$ is continuous.

We call an element $P \in \mathscr{F}_{1} \mathcal{A}$ elliptic if it is invertible and hence for all integers $n$ the element $\left[P^{n}\right] \in \mathcal{M}_{n}^{1}$ is also invertible in the associated graded algebra.

(4) There exists an elliptic element such that the map $\mathscr{F}_{n} \mathcal{A} / \widetilde{F}_{n-1} \mathcal{A} \ni\left[P^{n}\right] \rightarrow$ $P^{n} \in \widetilde{F}_{n} \mathcal{A}$ gives a linear splitting of $\widetilde{F}_{n-1} \mathcal{A} \hookrightarrow \widetilde{F}_{n} \mathcal{A} \rightarrow \widetilde{F}_{n} \mathcal{A} / \widetilde{F}_{n-1} \mathcal{A}$.

If $\mathcal{A}$ is a topologically filtered algebra then using the existence of an elliptic element, we have

$$
\begin{aligned}
& \widetilde{F}_{p} \mathcal{A}=\widetilde{F}_{p-1} \oplus \mathscr{F}_{p} \mathcal{A} / \mathscr{F}_{p-1} \mathcal{A} \\
& =\mathcal{F}_{p-2} \oplus \mathcal{F}_{p} \mathcal{A} / \mathcal{F}_{p-1} \mathcal{A} \oplus \mathcal{F}_{p-1} \mathcal{A} / \mathcal{F}_{p-2} \mathcal{A} \\
& =\tilde{F}_{p-2} \oplus \mathcal{F}_{p} \mathcal{A} / \widetilde{F}_{p-2} \mathcal{A} \text {. }
\end{aligned}
$$

Since $\mathcal{I}=0$, by repeating the iterations, we obtain

$$
\mathcal{F}_{p} \mathcal{A}=\lim _{\longleftarrow} \mathcal{F}_{p} \mathcal{A} / \mathcal{F}_{p-k} \mathcal{A}=\prod_{j \leq p} \mathcal{F}_{j} \mathcal{A} / \mathcal{F}_{j-1} \mathcal{A} .
$$

We use this description to endow $\mathscr{F}_{p} \mathcal{A}$ with the projective limit topology from the Fréchet topologies on $\mathscr{F}_{p} \mathcal{A} / \mathscr{F}_{p-k} \mathcal{A}$. $\mathcal{A}$ is then endowed with the inductive limit topology from $\mathcal{A}=\lim _{\longrightarrow} \mathcal{F}_{p} \mathcal{A}=\prod_{p} \mathcal{F}_{p} \mathcal{A} / \mathcal{F}_{p-1} \mathcal{A}$. Since strong inductive limits of nuclear spaces are nuclear, the topology on $\mathcal{A}$ is nuclear. We call the resultant topology the weak topology on $\mathcal{A}$. (The inductive limit topologies are 'strong' topologies. 
The nomenclature here is to distinguish the inductive limit topology on $\mathcal{A}$ from yet another topology which is stronger.) Since $\mathcal{A}$ is a filtered algebra, $\mathcal{A}_{0}:=\mathscr{F}_{0} \mathcal{A}$ is a subalgebra and further $\mathcal{A}_{-1}:=\mathscr{F}_{-1} \mathcal{A}$ is an ideal in $\mathcal{A}_{0}$. By property (4), each $A_{i}=\mathscr{F}_{i} \mathcal{A} / \mathscr{F}_{i-1} \mathcal{A}$ is a module over $A_{0}=\mathcal{A}_{0} / \mathcal{A}_{-1}$ generated by a single element $\left[P^{i}\right]$.

Proposition 6.2. The multiplication in $\mathcal{A}$ is separately continuous with respect to the weak topology. The multiplication is jointly continuous on the subalgebra $\mathcal{A}_{0}=$ $\mathcal{F}_{0} \mathcal{A}$.

Proof. First we prove that multiplication is jointly continuous on $\mathcal{A}_{0}$. This is an immediate consequence of the universal property of projective limits. Since the multiplication map on $\mathscr{F}_{0} \mathcal{A} / \mathscr{F}_{-j} \mathcal{A} \hat{\otimes} \mathscr{F}_{0} \mathcal{A} / \mathscr{F}_{-j} \mathcal{A} \rightarrow \mathscr{F}_{0} \mathcal{A} / \mathscr{F}_{-j} \mathcal{A}$ is continuous for each $j$, by composition there is a continuous map

$$
\phi_{j}: \lim _{\longleftarrow} \widetilde{F}_{0} \mathcal{A} / \mathcal{F}_{-j} \mathcal{A} \hat{\otimes} \lim _{\longleftarrow} \mathcal{F}_{0} \mathcal{A} / \widetilde{F}_{-j} \mathcal{A} \rightarrow \mathscr{F}_{0} \mathcal{A} / \mathcal{F}_{-j} \mathcal{A} .
$$

Using the universal property of $\lim _{\text {, }}$ there is thus a unique continuous map on $\phi: \lim \widetilde{F}_{0} \mathcal{A} / \mathscr{F}_{-j} \mathcal{A} \hat{\otimes} \lim \mathscr{F}_{0} \mathcal{A} / \overleftarrow{F}_{-j} \mathcal{A} \rightarrow \lim \mathscr{F}_{0} \mathcal{A} / \mathscr{F}_{-j} \mathcal{A}=\mathcal{A}_{0}$. To complete

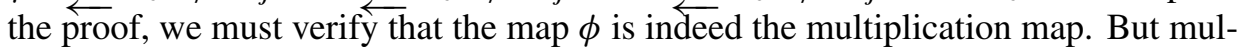
tiplication on $\mathcal{A}_{0}$ composed with the projection $\mathcal{A}_{0} \rightarrow \mathscr{F}_{0} \mathcal{A}_{/} / \mathscr{F}_{-1} \mathcal{A}$ is just the map $\phi_{j}$ above and hence the multiplication must be $\phi$ by uniqueness (as algebraic maps) on the projective limit:

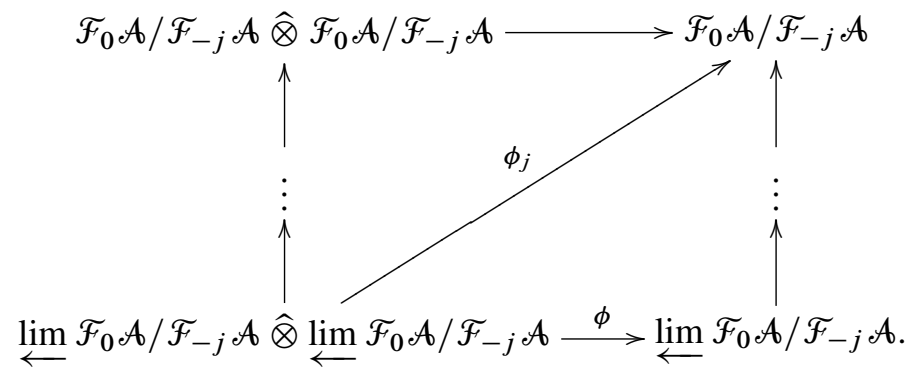

The separate continuity of the multiplication on $\mathcal{A}$ can be proved similarly.

Although a topologically filtered algebra $\mathcal{A}$ is a nuclear space and the completed projective tensor product $\mathcal{A}^{\hat{\otimes} n}$ is again nuclear by Proposition 6.2, the Hochschild boundary map $b$ may not be continuous on $\mathscr{A}^{\widehat{\otimes} n}$. We can define a Hochschild complex for $\mathcal{A}$ in the following fashion.

Let

$$
\mathscr{F}_{p}^{\prime}:=\sum_{\sum p_{i} \leq p} \widetilde{F}_{p_{0}} \mathcal{A} \hat{\otimes} \mathscr{F}_{p_{1}} \mathcal{A} \hat{\otimes} \ldots \hat{\otimes} \mathscr{F}_{p_{n}} \mathcal{A} .
$$

Let $\mathscr{F}_{p} C_{n}(\mathcal{A}):=\lim _{\overleftarrow{F^{\prime}}} \widetilde{F}_{p}^{\prime} / \mathscr{F}_{p-f}^{\prime}$. Then the differentials $b$ and $b^{\prime}$ are continuous on each filtration, and if $1 \in \mathscr{F}_{0} \mathcal{A}$, then the operators $s, t, N$ and $B$ induce continuous 
maps on $\mathscr{F}_{p} C_{n}(\mathcal{A})$. Denote by $\mathscr{F}_{p} \mathrm{HH}_{*}(\mathcal{A})$ the homology of the complex $\mathscr{F}_{p} C_{*}(\mathcal{A})$. We define the Hochschild homology of $\mathcal{A}$ to be $\operatorname{HH}_{*}(\mathcal{A})=\lim \widetilde{F}_{p} \mathrm{HH}_{*}(\mathcal{A})$.

Since it is often useful to use spectral sequences to compute homologies for such filtered algebras, it is convenient to describe the topology on the associated graded algebra of $\mathcal{A}$ by using the identification

$$
\operatorname{Gr}(\mathcal{A})=\lim _{p=-N} \bigoplus_{p}^{N} \mathcal{F}_{p} \mathcal{A} / \mathcal{F}_{p-1} \mathcal{A} .
$$

Moreover, the topology on the Hochschild complex for $\operatorname{Gr}(\mathcal{A})$ is defined by

$$
\mathscr{H}_{n}(\operatorname{Gr}(\mathcal{A})):=\lim _{\longrightarrow}\left(\bigoplus_{p=-N}^{N} \mathcal{F}_{p} \mathcal{A} / \mathscr{F}_{p-1} \mathcal{A}\right)^{\otimes n+1} .
$$

Again the Hochschild boundary map is continuous with respect to this topology. The homogeneous components of $\operatorname{Gr}(\mathcal{A})$ can be defined by

$$
\mathscr{H}_{n}(\operatorname{Gr}(\mathcal{A}))_{p}:=\lim _{p=-N} \bigoplus_{p={ }_{k_{j}}}^{N} \hat{F}_{k_{j}} \mathcal{A} / \mathcal{F}_{k_{j}-1} \mathcal{A},
$$

where $-N \leq k_{j} \leq N$ and $\sum k_{i} \leq p$.

Our main examples of topologically filtered algebras are the algebra of complete symbols $\mathcal{A}(M)$ over a closed manifold $M$ and its cross-product $\mathcal{A}(M) \rtimes \Gamma$ with a finite group $\Gamma$. Other examples include the Heisenberg complete symbol algebra $\mathcal{A}_{H}(M)$ over a Heisenberg manifold $(M, H)$.

\section{Symplectic Poisson structure}

To compute the homology of $\mathscr{B}(M):=\mathcal{A}(M) \rtimes \Gamma$ we shall need a few basic facts about the canonical symplectic structure on the $T^{*} M \backslash\{0\}$ cotangent bundle minus the zero section. First of all we observe that for an element $\gamma \in \Gamma$ the fixed point set $T^{*} M^{\gamma}$ is symplectic and can be identified as a symplectic manifold with $T^{*}\left(M^{\gamma}\right)$. (The same holds with the zero section removed.) Further one can choose an extension procedure that assigns to a smooth function $f \in \mathcal{C}^{\infty}\left(T^{*} M^{\gamma} \backslash\{0\}\right)$ a function $\tilde{f} \in \mathcal{C}^{\infty}\left(T^{*} M \backslash\{0\}\right)$ satisfying the following:

(i) The procedure is a symplectic extension as described in Proposition 7.1 below.

(ii) The procedure is $\Gamma_{\gamma}$ equivariant.

The existence of such a procedure is easy to establish.

Proposition 7.1. Let $Y$ be a symplectic submanifold of $X$. Then there exists an extension $C^{\infty}(Y) \rightarrow C^{\infty}(X)$ denoted by $f \rightarrow \hat{f}$ such that $\{\hat{f}, \hat{g}\}_{\left.X\right|_{Y}}=\{f, g\}_{Y}$ for all $f, g \in C^{\infty}(Y)$. 
We call an extension $C^{\infty}(Y) \rightarrow C^{\infty}(X)$ with the above property a symplectic extension.

Proof. By choosing a connection on the bundle $T Y^{\perp} \stackrel{\pi_{\perp}}{\longrightarrow} Y$ we can obtain a symplectic form $\omega_{\perp}$ on $T Y^{\perp}$ such that $Y$ is a symplectic submanifold and extending a function $f \in \mathcal{C}^{\infty}(Y)$ to $\pi_{\perp}^{*} f$ produces

$$
\left\{\pi_{\perp}^{*} f, \pi_{\perp}^{*} g\right\}_{\left.T Y\right|_{Y} ^{\perp}}=\{f, g\}_{Y} \text { for all } f, g \in C^{\infty}(Y) .
$$

By identifying a small enough tubular neighbourhood $U_{0}$ of $Y$ in $X$ with a neighbourhood of the zero section $U_{1}$ in $T Y^{\perp}$ we find a Moser diffeomorphism $\phi: U_{0} \rightarrow U_{1}$ such that $\phi^{*} \omega_{\perp}=\omega$. The required extension is $\hat{f}$ is any extension of the function $\phi^{*}\left(\left.\pi_{\perp}^{*} f\right|_{U_{0}}\right)$ using some partition of unity.

7.1. Canonical homology of symplectic manifolds. The Poisson structure on any Poisson manifold $M$ can be used to define a differential on differential forms,

$$
\delta: \Omega^{k}(M) \rightarrow \Omega^{k-1}(M),
$$

and has a local expression of he form

$$
\begin{aligned}
\delta\left(f_{0} d f_{1} \wedge \cdots \wedge d f_{k}\right)= & \sum_{1 \leq i \leq k}(-1)^{i-1}\left\{f_{0}, f_{i}\right\}_{M} d f_{1} \wedge \cdots \wedge \widehat{d f_{i}} \cdots \wedge d f_{k} \\
& +\sum_{1 \leq i<j \leq k}(-1)^{i+j-1} f_{0} d\left\{f_{i}, f_{j}\right\}_{M} \wedge d f_{1} \wedge \cdots \\
& \cdots \wedge \widehat{d f_{i}} \wedge \ldots \wedge \wedge \widehat{d f_{j}} \wedge \cdots \wedge d f_{k} .
\end{aligned}
$$

The complex $\left(\Omega^{*}(M), \delta\right)$ is called the Koszul complex of the Poisson manifold $(M,\{\})$, and its homology the Poisson homology, which we denote by $H K_{*}(M)$.

Although $\delta$ can be defined for any Poisson manifold, we will restrict to the case of the symplectic manifold $M$ of dimension $2 n$. Here one can define a sympletic $*$ operator $*: \Omega^{*}(M) \rightarrow \Omega^{*} 2 n-*(M)$. The $\delta, d$ and $*$ satisfy the following relations [6]:

(1) If $\alpha \in \Omega^{k}(X)$, then $* * \alpha=\alpha$.

(2) $\delta=(-1)^{k+1} * d *$ on $\Omega^{k}(X)$.

(3) $d \delta+\delta d=0$ as a map on $\Omega^{k}(X)$.

A direct consequence is the following result for canonical homology of symplectic manifolds..

Proposition 7.2. Let $(M, \omega)$ be a compact symplectic manifold of dimension $2 n$. Then $(-1)^{k+1} *$ is a chain map between $\left(\Omega^{k}(X), \delta\right)$ and $\left(\Omega^{2 n-k}(X), d\right)$, and the symplectic Poisson homology is given by

$$
H K_{k}(M)=H_{\text {deRham }}^{2 n-k}(M) \text {. }
$$


Now let $X=T^{*} M \backslash\{0\}$ be the cotangent bundle minus the zero section. Then there is an $\mathbb{R}^{+}$action on $X$. Let $\Xi$ be the Euler vector field on $X=T^{*} M \backslash\{0\}$ generated by the action of $\mathbb{R}^{+}$. The canonical one form on $X$ is the form $\alpha=i(\Xi) \omega$. Let $\varepsilon(\alpha): \Omega^{k}(X) \rightarrow \Omega^{k+1}(X)$ be the exterior multiplication by $\alpha$. The following analogue of Cartan's magic formula provides us with necessary contractions. (See also [7].)

Lemma 7.3. Let $\mathscr{L}_{Y}$ denote the Lie derivative with respect to a vector field $Y$. The following identity holds on $k$-forms $\Omega^{k}$ :

$$
\delta \varepsilon(\alpha)+\varepsilon(\alpha) \delta=\mathscr{L}_{\Xi}+n-k .
$$

In particular, if $\beta$ is a $k$-form which is homogeneous of degree $l$, then $\mathscr{L}_{\Xi} \beta=l \beta$ and therefore $(\delta \varepsilon(\alpha)+\varepsilon(\alpha)) \beta=l+n-k$. The differential $\delta$ occurs in the spectral sequence calculation below and Lemma 7.3 provides a contraction.

\section{Spectral sequence for the cross-product}

The action of the group $\Gamma$ preserves the natural filtration on the complete symbol algebra $\mathcal{A}(M)$ and hence provides the crossproduct algebra $\mathscr{B}(M)$ with a natural filtration $\mathscr{F}_{i} \mathscr{B}(M)=\mathscr{F}_{i} \mathcal{A}(M) \rtimes \Gamma$. Let $P$ be a positive elliptic operator invariant under the $\Gamma$ action. Let $\rho$ be the symbol for $P$. The associated graded algebra then can be identified as

$$
\begin{aligned}
\operatorname{Gr}(\mathcal{B}(M)) & \simeq \bigoplus_{l \in \mathbb{Z}} e^{\infty}\left(T^{*} M \backslash\{0\}\right)_{l} \rtimes \Gamma \\
& \simeq \bigoplus_{l \in \mathbb{Z}}\left(\bigodot^{\infty}\left(S^{*} M\right) \rtimes \Gamma\right) \rho^{l} \simeq \bigodot^{\infty}\left(S^{*} M\right) \rtimes \Gamma \otimes \mathbb{C}\left[\rho, \rho^{-1}\right] .
\end{aligned}
$$

(Here and elsewhere $\dot{\ell}^{\infty}(Z)$ and $\dot{\Omega}^{k}(Z)_{l}$ represent homogeneous sections of degree $l$ on a principal $\mathbb{R}^{+}$bundle $Z$.) With this filtration the $E^{1}$-term in the Hochschild homology spectral sequence of $\mathscr{B}(M)$ is

$$
E^{1}=\mathrm{HH}_{*}(\operatorname{Gr}(\mathscr{B}(M))) \simeq \mathrm{HH}_{*}\left(\left(\mathcal{C}^{\infty}\left(S^{*} M\right) \rtimes \Gamma\right) \otimes \mathbb{C}\left[\rho, \rho^{-1}\right]\right),
$$

which, by Theorem 4.6, can be identified as $E^{1}=\bigoplus_{\langle\gamma\rangle} \dot{\Omega}^{*}\left(T^{*} M \backslash\{0\}^{\gamma}\right)^{\Gamma_{\gamma}}=$ $\bigoplus_{\langle\gamma\rangle} E_{\gamma}^{1}$, where $\gamma$ runs over a set of representatives of conjugacy classes. The theorem below describes the $d^{1}$ differential under this identification.

Theorem 8.1. For every $k \geq 0$, we have the diagram

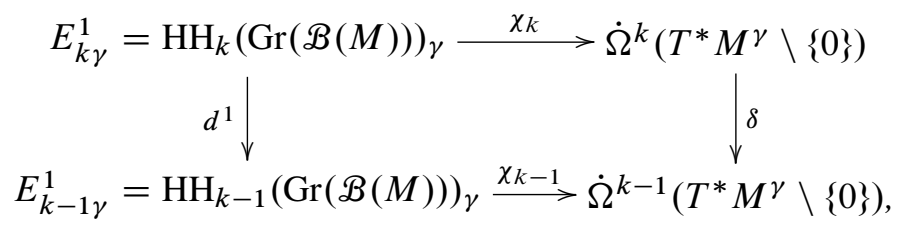


where the differential $\delta$ is given by the equation (5) on $T^{*} M^{\gamma}$.

Proof. Let $f_{i} \in \mathcal{C}^{\infty}\left(T^{*} M^{\gamma} \backslash\{0\}\right), 0 \leq i \leq k$, be homogeneous functions of degree $p_{i}$ and $\Gamma_{\gamma}$ invariant. Let $p=p_{0}+p_{1}+\cdots+p_{k}$. Also, let

$$
\xi=f_{0} d f_{1} d f_{2} \ldots d f_{k} \in \dot{\Omega}^{k}\left(T^{*} M^{\gamma} \backslash\{0\}\right) .
$$

Then the $k$-form $\xi$ is homogeneous of degree $\mathrm{p}$, and since it is $\Gamma_{\gamma}$ invariant by the isomorphism (6), it gives a class in $\mathrm{HH}_{k}\left(\mathcal{C}^{\infty}\left(T^{*} M \backslash\{0\}\right)\right)_{\gamma}$ represented by

$$
E_{k}(\xi)=\sum_{\pi \in S_{k}} \epsilon(\pi) \tilde{f}_{0} \gamma \otimes \tilde{f}_{\pi(1)} e \otimes \tilde{f}_{\pi(2)} e \cdots \otimes \tilde{f}_{\pi(k)} e,
$$

where $\tilde{f}_{i}$ is a symplectic extension of $f_{i}$. Since $\Gamma_{\gamma}$ actions on $T^{*} M \backslash\{0\}$ are through symplectomorphisms, the extensions $\tilde{f}_{i}$ are in fact invariant extensions of $f_{i}$ to the whole of $T^{*} M \backslash\{0\}$. We only have to check that $\delta=\chi \circ d^{1} \circ E$. To evaluate $d^{1}$ on $\mathrm{HH}_{*}(\operatorname{Gr}(\mathscr{B}(M)))_{\gamma}$, we must lift the form $\xi$ to a tensor. We now choose operators $A_{0}, A_{1}, \ldots A_{k} \in \mathscr{F}_{p_{i}} \mathcal{A}(M)=\Psi^{p_{i}}(M) / \Psi^{-\infty}(M)$, which are $\Gamma_{\gamma}$ invariant and such that $\sigma\left(A_{j}\right)=\tilde{f}_{j}$. (This can be done by averaging on a $\Gamma$ equivariant splitting of $\sigma$.) Using the above choice of lifting,

$$
\sigma^{-1}\left(E_{k}(\xi)\right)=\sum_{\pi \in S_{k}} \epsilon(\pi) A_{0} \gamma \otimes A_{\pi(1)} e \otimes A_{\pi(2)} e \cdots \otimes A_{\pi(k) e} .
$$

On applying $b$ the Hochschild differential, there are three different kinds of expressions for each $\pi \in S_{k}$. So the resultant expression can be broken up as the sum of

$$
\begin{aligned}
\boldsymbol{A} & =\sum_{\pi \in S_{k}} \epsilon(\pi) A_{0} A_{\pi(1)} \gamma \otimes A_{\pi(2)} e \otimes \cdots \otimes A_{\pi(k)} e, \\
\boldsymbol{B} & =\sum_{\pi \in S_{k}} \sum_{i=1}^{k-1}(-1)^{-1} \epsilon(\pi) A_{0} \gamma \otimes \cdots \otimes A_{\pi(i)} A_{\pi(i+1)} e \otimes \cdots \otimes A_{\pi(k)} e, \\
\boldsymbol{C} & =\sum_{\pi \in S_{k}}(-1)^{k} \epsilon(\pi) A_{\pi(k)} A_{0} \gamma \otimes A_{\pi(1)} e \otimes \cdots \otimes A_{\pi(k-1)} e .
\end{aligned}
$$

(Remember that $A_{i}$ 's have been chosen to be invariant under $\gamma$.) By replacing $\pi$ in $\boldsymbol{A}$ by $\pi \tau$ where $\tau$ is a cyclic permutation, we can rewrite it as

$$
\boldsymbol{A}=\sum_{\pi \in \mathcal{S}_{k}}(-1)^{k+1} \epsilon(\pi) A_{0} A_{\pi(k)} \gamma \otimes A_{\pi(1)} e \otimes \cdots \otimes A_{\pi(k-1)} e .
$$

Thus

$$
\boldsymbol{A}+\boldsymbol{C}=\sum_{\pi \in \mathcal{S}_{k}}(-1)^{k+1} \epsilon(\pi)\left\{A_{0}, A_{\pi(k)}\right\} \gamma \otimes A_{\pi(1)} e \otimes \cdots \otimes A_{\pi(k-1)} e,
$$


or in $\mathrm{HH}_{*}(\operatorname{Gr}(\mathscr{B}(M))$ it is represented by

$$
\sum_{\pi \in S_{k}}(-1)^{k+1} \epsilon(\pi)\left\{f_{0}, f_{\pi(k)}\right\} \gamma \otimes f_{\pi(1)} e \otimes \cdots \otimes f_{\pi(k-1)} e .
$$

For any permutation $\pi$ for which $\pi(k)=i$ is fixed, the image of the tensor

$$
(-1)^{k+1} \epsilon(\pi)\left\{f_{0}, f_{\pi(k)}\right\} \gamma \otimes f_{\pi(1)} e \otimes \cdots \otimes f_{\pi(k-1)} e
$$

under $\chi$ of Theorem 6 is the same, namely

$$
\frac{1}{k-1 !}(-1)^{i}\left\{f_{0}, f_{i}\right\} d f_{1} d f_{2} \ldots \widehat{d f}_{i} \ldots d f_{k} .
$$

(Again remember that all the $f_{j}$ 's are $\Gamma_{\gamma}$ invariant.) There are $(k-1)$ ! permutations such that $\pi(k)=i$ for fixed $i$, and therefore the parts corresponding to $\boldsymbol{A}$ and $\boldsymbol{C}$ in $d^{1}$ become

$$
\sum_{1 \leq i \leq k}(-1)^{i-1}\left\{f_{0}, f_{i}\right\} d f_{1} \wedge \cdots \wedge d f_{i} \cdots \wedge d f_{k} .
$$

The summand $\boldsymbol{B}$ pairs each $\pi$ with the transpositions $\pi(i i+1)$,

$$
\boldsymbol{B}=\frac{1}{2} \sum_{\pi \in S_{k}} \sum_{i=1}^{k-1}(-1)^{-1} \epsilon(\pi) A_{0} \gamma \otimes \cdots \otimes\left\{A_{\pi(i)}, A_{\pi(i+1)}\right\} e \otimes \cdots \otimes A_{\pi(k)} e,
$$

and so $\sigma(\boldsymbol{B})=\frac{1}{2} \sum_{\pi \in S_{k}} \sum_{i=1}^{k-1}(-1)^{i-1} \epsilon(\pi) f_{0} \gamma \otimes \cdots \otimes\left\{f_{\pi(i)}, f_{\pi(i+1)}\right\} e \otimes \cdots \otimes$ $f_{\pi(k)} e$. All pairs $(\pi, i)$ such that the set $\{\pi(i), \pi(i-1)\}$ is the same as the set $\{m, n\}$, $m<n$ have the same image under $\chi$, namely the form

$$
\frac{(-1)^{m+n}}{k-1} f_{0} d\left\{f_{m}, f_{n}\right\} \wedge d f_{1} \wedge \cdots \wedge d \hat{f}_{m} \wedge \cdots \wedge \hat{d f_{n}} \wedge \cdots \wedge d f_{k} .
$$

As there are $2(k-1)$ ! such pairs for each $\{m, n\}$, the terms in $\boldsymbol{B}$ map to the remainder of $\delta$

$$
\sum_{1 \leq i<j \leq k}(-1)^{i+j-1} f_{0} d\left\{f_{i}, f_{j}\right\} \wedge d f_{1} \wedge \ldots \hat{d f_{i}} \wedge \ldots \hat{d f_{j}} \wedge \ldots \wedge d f_{k}
$$

As corollaries we obtain all our main results.

Corollary 8.2. We have

$$
\mathrm{HH}_{*}(\mathcal{A}(M) \rtimes \Gamma)=\sum_{\langle\gamma\rangle} H^{2 k_{\gamma}-*}\left(S^{*} M^{\gamma} \times S^{1}\right)^{\Gamma_{\gamma}},
$$

where $k_{\gamma}=\operatorname{dim}\left(M^{\gamma}\right)$. 
Proof. Since the $\Gamma_{\gamma}$ action on $T^{*} M^{\gamma} \backslash\{0\}$ is by symplectomorphisms, all $\gamma \in \Gamma$ commute with the symplectic $*$ operator and therefore with the operator $\delta$. Hence by Theorem 4.6 and the previous proposition, $E_{k \gamma}^{2} \simeq H^{2 k_{\gamma}-*}\left(S^{*} M^{\gamma} \times S^{1}\right)^{\Gamma_{\gamma}}$.

We now observe that the differential $d^{2}$ for this spectral sequence vanishes. The $E_{\gamma}^{1}$ can be given a $\mathbb{Z}$ bigrading with $E_{\gamma, k l}^{1}$ being $k$-forms of homogeneity $l$ on $T^{*}\left(M^{\gamma} \backslash\right.$ $\{0\})$. Then the differential $d^{1}=\delta$ maps $E_{\gamma, k l}^{1} \rightarrow E_{\gamma, k-1 l-1}^{1}$. This is because the Poisson bracket decreases the homogeneity by 1 . But on $E_{\gamma, k l}^{1}$, by 7.3, we have

$$
\delta \varepsilon(\alpha)+\varepsilon(\alpha) \delta=\mathscr{L}_{\Xi}+k_{\gamma}-k=l+k_{\gamma}-k .
$$

Thus unless $l=k-k_{\gamma}$ there is a contraction for $\delta$ on $E_{\gamma, k l}^{1}$. Therefore the only nonzero terms on $E_{k l}^{2}$ correspond to $l=k_{\gamma}-k$. The differential $d^{2}$ must thus either start or end in a 0 -term.

Since the Hochschild homology groups are finite dimensional, the Hochschild cohomology groups are the dual of the Hochschild homology groups. Hence by Poincaré duality the rank $\mathrm{HH}^{0}(\mathscr{B}(M))$ is the number of path components of $S^{*} M^{\gamma}$ where $\gamma$ ranges over all conjugacy classes.

To compute the cyclic homology, we use Connes' SBI exact sequence:

$$
\begin{aligned}
\ldots \stackrel{B}{\rightarrow} \mathrm{HH}_{n}(\mathcal{B}(M)) & \stackrel{I}{\rightarrow} \mathrm{HC}_{n}(\mathcal{B}(M)) \stackrel{S}{\rightarrow} \mathrm{HC}_{n-2}(\mathcal{B}(M)) \\
& \stackrel{B}{\rightarrow} \mathrm{HH}_{n-1}(\mathcal{B}(M)) \rightarrow \cdots .
\end{aligned}
$$

Here the connecting morphism $B: \operatorname{Tot}\left(B_{*}(\mathscr{B}(M))\right) \rightarrow \mathscr{H}_{*}(\mathscr{B}(M))[-1]$ is the cyclic boundary map $B$.

Proposition 8.3. The connecting morphism $B$ in the SBI exact sequence for $\mathscr{B}(M)$ vanishes and hence

$$
\mathrm{HC}_{k}(\mathscr{B}(M))=\sum_{j \geq 0} \mathrm{HH}_{k-2 j}(\mathscr{B}(M)) .
$$

Proof. Since $\mathcal{B}(M)$ is unital, $B$ is induced from a chain map

$$
\operatorname{Tot}\left(B_{*}(\mathscr{B}(M))\right) \rightarrow \mathscr{H}_{*}(\mathscr{B}(M))[-1]
$$

that respects the filtration on both the source and the range complex. Thus $B$ induces a map on the spectral sequence $\mathrm{EC}^{*}$ of $\operatorname{Tot}\left(B_{*}(\mathscr{B})\right)$ into the spectral sequence $\mathrm{EH}^{*}$ of $\mathscr{H}_{*}(\mathscr{B}(M))[-1]$. The induced map on $\mathrm{EC}^{1} \rightarrow \mathrm{EH}^{1}$ when identified with the space of differential forms is the de Rham differential $d$ which vanishes on $E^{2}$ :

$$
d: \mathrm{EC}_{k, l}^{1}=\bigoplus_{j} \Omega^{k-2 j}\left(S^{*} M^{\gamma} \times S^{1}\right)_{l}^{\Gamma_{\gamma}} \rightarrow \mathrm{EH}_{k, l}^{1}=\Omega^{k+1}\left(S^{*} M^{\gamma} \times S^{1}\right)_{l}^{\Gamma_{\nu}} .
$$

For the EC-term to contribute to nonzero homology, $l=2 k_{\gamma}-k$, whereas for the EH-term $l=2 k_{\gamma}-k-1$.

The formula for $\mathrm{HC}_{k}(\mathscr{B})$ then follows from the SBI sequence. 
8.1. Modules of Fourier integral operators. In the case of the algebra $\mathcal{A}(M)$ and a diffeomorphism $h$ on the manifold $M$ the module $\mathcal{A}(M)_{h}$ as described in Lemma 4.1 is the module of Fourier integral operators corresponding to the canonical relation being the conormal bundle $\mathfrak{N}^{*}$ graph $(h)$ with classical symbols. Thus in view of (3) one immediately obtains

$$
\mathrm{HH}_{*}\left(\mathcal{A}(M), \mathcal{A}(M)_{h}\right) \simeq H^{2 k_{h}-*}\left(S^{*} M^{h} \times S^{1}\right) .
$$

One natural question is to consider more general modules of Fourier Integral operators over $\mathcal{A}(M)$ and investigate the corresponding Hochschild and cyclic homology. This requires a slightly different approach than the one described here and is presently work in progress. But for now we have already proved the following.

Proposition 8.4. Let $h: M \rightarrow M$ be a diffeomorphism such that $S^{*} M^{h}$ is a connected smooth manifold. Then there exists (up to a constant) a unique twisted trace $\tau_{h}: \mathcal{A}(M) \rightarrow \mathbb{C}$ such that

$$
\tau_{h}(A \cdot B)=\tau_{h}(B \cdot h(A))
$$

The existence is already clear from (1) and uniqueness follows from the above observation.

\section{References}

[1] B. Ammann, R. Lauter, V. Nistor, and A. Vasy, Complex powers and non-compact manifolds. Comm. Partial Differential Equations 29 (2004), 671-705. Zbl 1071.58022 MR 2059145

[2] M. F. Atiyah and R. Bott, A Lefschetz fixed point formula for elliptic complexes: I. Ann. of Math. (2) 86 (1967), 374-407. Zbl 0161.43201 MR 0212836

[3] P. Baum and A. Connes, $K$-theory for discrete groups. In Operator algebras and applications, Vol. 1, London Math. Soc. Lecture Note Ser. 135, Cambridge University Press, Cambridge 1988, 1-20. Zbl 0685.46041 MR 996437

[4] M.-T. Benameur and V. Nistor, Homology of algebras of families of pseudodifferential operators. J. Funct. Anal. 205 (2003), 1-36. Zbl 1048.47052 MR 2020206

[5] J. Brüning and E. Heintze, Representations of compact Lie groups and elliptic operators. Invent. Math. 50 (1979), 169-203. Zbl 0392.58015 MR 517776

[6] J.-L. Brylinski, A differential complex for Poisson manifolds. J. Differential Geom. 28 (1988), 93-114. Zbl 0634.58029 MR 950556

[7] J.-L. Brylinski and E. Getzler, The homology of algebras of pseudo-differential symbols and the noncommutative residue. K-Theory 1 (1987), 385-403. Zbl 0646.58026 MR 920951

[8] J.-L. Brylinski and V. Nistor, Cyclic cohomology of étale groupoids. K-Theory 8 (1994), 341-365. Zbl 0812.19003 MR 1300545 
[9] A. Connes, Noncommutative geometry. Academic Press, San Diego, CA, 1994. Zbl 0818.46076 MR 1303779

[10] A. Connes and H. Moscovici, The local index formula in noncommutative geometry. Geom. Funct. Anal. 5 (1995), 174-243. Zbl 0960.46048 MR 1334867

[11] P. B. Gilkey, Invariance theory, the heat equation, and the Atiyah-Singer index theorem. 2nd ed., CRC Press, Boca Raton, FL, 1995. Zbl 0856.58001 MR 1396308

[12] V. Guillemin, A new proof of Weyl's formula on the asymptotic distribution of eigenvalues. Adv. in Math. 55 (1985), 131-160. Zbl 0559.58025 MR 772612

[13] M. Kontsevich and S. Vishik, Geometry of determinants of elliptic operators. In Functional analysis on the eve of the 21 st century, Vol. I, Progr. Math. 131, Birkhäuser, Boston 1995, 173-197. Zbl 0920.58061 MR 1373003

[14] J. Korevaar, A century of complex Tauberian theory. Bull. Amer. Math. Soc. (N.S.) 39 (2002), 475-531. Zbl 1001.40007 MR 1920279

[15] R. Lauter and S. Moroianu, Homology of pseudo-differential operators on manifolds with fibered boundaries. J. Reine Angew. Math. 547 (2002), 207-234. Zbl 0994.58017 MR 1900142

[16] R. Lauter and S. Moroianu, Homology of pseudodifferential operators on manifolds with fibered cusps. Trans. Amer. Math. Soc. 355 (2003), 3009-3046. Zbl 1024.58012 MR 1974673

[17] M. Lesch, On the noncommutative residue for pseudodifferential operators with log-polyhomogeneous symbols. Ann. Global Anal. Geom. 17 (1999), 151-187. Zbl 0920.58047 MR 1675408

[18] J.-M. Lescure and S. Paycha, Uniqueness of multiplicative determinants on elliptic pseudodifferential operators. Proc. Lond. Math. Soc. (3) 94 (2007), 772-812. Zbl 1193.58018 MR 2325320

[19] J.-L. Loday, Cyclic homology. 2nd ed., Grundlehren Math. Wiss. 301, Springer-Verlag, Berlin 1998. Zbl 0885.18007 MR 1600246

[20] S. Mac Lane, Homology. Classics Math., Springer-Verlag, Berlin 1995. Zbl 0818.18001 MR 1344215

[21] S. Moroianu, Weyl laws on open manifolds. Preprint 2003. arXiv:math/0310075

[22] S. Moroianu and V. Nistor, Index and homology of pseudodifferential operators on manifolds with boundary. In Perspectives in operator algebras and mathematical physics, Theta Ser. Adv. Math. 8, Theta, Bucharest 2008, 123-148. Zbl 1212.58017 MR 2433031

[23] V. Nistor, Group cohomology and the cyclic cohomology of crossed products. Invent. Math. 99 (1990), 411-424. Zbl 0692.46065 MR 1031908

[24] E. Schrohe, Complex powers of elliptic pseudodifferential operators. Integral Equations Operator Theory 9 (1986), 337-354. Zbl 0597.35123 MR 846534

[25] E. Schrohe, Noncommutative residues and manifolds with conical singularities. J. Funct. Anal. 150 (1997), 146-174. Zbl 0903.58057 MR 1473629

[26] R. T. Seeley, Complex powers of an elliptic operator. In Singular integrals (Proc. Sympos. Pure Math., Chicago, Ill., 1966), Amer. Math. Soc., Providence, R.I., 1967, 288-307. Zbl 0159.15504 MR 0237943 
[27] M. Wodzicki, Noncommutative residue. I. Fundamentals. In $K$-theory, arithmetic and geometry (Moscow, 1984-1986), Lecture Notes in Math. 1289, Springer, Berlin 1987, 320-399. Zbl 0649.58033 MR 923140

[28] M. Wodzicki, Excision in cyclic homology and in rational algebraic $K$-theory. Ann. of Math. (2) 129 (1989), 591-639. Zbl 0689.16013 MR 997314

Received January 17, 2011; revised June 22, 2011

S. Dave, Fakultät für Mathematik, Universität Wien, Oskar-Morgenstern-Platz 1, 1090 Wien, Austria

E-mail: shantanu.dave@univie.ac.at 